TRANSACTIONS OF THE

AMERICAN MATHEMATICAL SOCIETY

Volume 348, Number 12, December 1996, Pages 4737-4773

S 0002-9947(96)01555-3

\title{
ON THE GROUP OF HOMOTOPY EQUIVALENCES OF A MANIFOLD
}

\author{
HANS JOACHIM BAUES
}

\begin{abstract}
We consider the group of homotopy equivalences $\mathcal{E}(M)$ of a simply connected manifold $M$ which is part of the fundamental extension of groups due to Barcus-Barratt. We show that the kernel of this extension is always a finite group and we compute this kernel for various examples. This leads to computations of the group $\mathcal{E}(M)$ for special manifolds $M$, for example if $M$ is a connected sum of products $S^{n} \times S^{m}$ of spheres. In particular the group $\mathcal{E}\left(S^{n} \times S^{n}\right)$ is determined completely. Also the connection of $\mathcal{E}(M)$ with the group of isotopy classes of diffeomorphisms of $M$ is studied.
\end{abstract}

The group $\mathcal{E}(X)$ of homotopy equivalences of a space $X$ is the set of homotopy classes of homotopy equivalences $X \rightarrow X$. The group structure is induced by mapcomposition. The group $\mathcal{E}(X)$, i.e. the group of automorphisms of the homotopy type of $X$, can be regarded as the homotopy symmetry group of the space $X$. In the literature there has been a lot of interest in the computation of such groups; compare for example the excellent survey article of M. Arkowitz [2].

This paper is concerned with the structure of $\mathcal{E}(M)$ in case $M$ is a closed, compact, oriented manifold, or more generally a Poincaré-complex. The computation of this group is an important step for the diffeomorphism classification of manifolds by surgery, [40]. The group $\mathcal{E}(M)$ is also important for Cooke's theory [17] of replacing homotopy actions by actions. For a differential manifold $M$ the group $\mathcal{E}(M)$ is comparable with the group $\Pi_{0} \operatorname{Diff}(M)$ of isotopy classes of diffeomorphisms of $M$. In fact, via the $\mathcal{J}$-homomorphism there is a striking similarity between these groups as is shown in $\S 10$ below.

Still there is little known on the group $\mathcal{E}(M)$ in the literature; only very specific examples are computed, see [2]. This paper contains on the one hand general results on the structure of the group $\mathcal{E}(M)$, see $\S 1, \ldots, \S 5$; on the other hand our methods are used for explicit computations, see $\S 6, \ldots, \S 10$.

Let $e$ be a small open cell of the simply connected $m$-dimensional manifold $M$ and let $\dot{M}=M-e$ be the complement. The inclusion $f: S^{m-1} \subset \dot{M}$ of the boundary is the attaching map for $M$. We consider the fundamental extension of groups due to Barcus-Barratt [42],

$$
\mathcal{E}(M \mid \dot{M}) \longmapsto \mathcal{E}(M) \rightarrow \mathcal{E}(\dot{M}, \pm f) .
$$

Here $\mathcal{E}(M \mid \dot{M})$ is the subgroup of $\mathcal{E}(M)$ consisting of all elements which can be represented by an orientation preserving map $M \rightarrow M$ under $\dot{M}$. This group is

Received by the editors August 17, 1994.

1991 Mathematics Subject Classification. Primary 55O10, 57 O50.

(c)1996 American Mathematical Society 
abelian and an $\mathcal{E}(\dot{M}, \pm f)$-module where $\mathcal{E}(\dot{M}, \pm f)$ is the group of elements in $\mathcal{E}(\dot{M})$ compatible with the attaching map up to sign.

Theorem (1.4). For a simply connected Poincaré-complex $M$ the abelian group $\mathcal{E}(M \mid \dot{M})$ is finite.

We now describe examples of fundamental extensions. For a product of spheres, $S^{n} \times S^{n}$, we get

Theorem (6.3). For $n \geq 1$ one has the split extension of groups

$$
T_{n} \oplus T_{n} \longmapsto \mathcal{E}\left(S^{n} \times S^{n}\right) \rightarrow G_{n}
$$

where $T_{n}$ is the torsion subgroup of $\Pi_{2 n+1} S^{n+1}$ and where $G_{n}$ is the subgroup of $G l_{2}(\mathbb{Z})$ in (6.1). The direct sum $T_{n} \oplus T_{n}$ is a $G_{n}$-module in a canonical way, see (6.3). In (6.2) the group $T_{n}$ is listed for $n \leq 10$.

Various authors worked on the group $\mathcal{E}\left(S^{n} \times S^{n}\right)$ but this result, for the first time, determines the group completely, see $\S 6$. In (6.5) we consider the groups $\mathcal{E}\left(S^{m} \times S^{n}\right)$ for $m>n$. A computation of such groups is achieved for certain products $S^{m} \times S^{n}$, see Theorem (6.7). In fact, we consider the $q$-fold connected sum

$$
q S^{m} \times S^{n}=\left(S^{m} \times S^{n}\right) \# \cdots \#\left(S^{m} \times S^{n}\right)
$$

and describe the fundamental extension for $\mathcal{E}\left(q S^{m} \times S^{n}\right)$ for $2 \leq n<m \leq 2 n-2$, $q \geq 1$, in Theorem (7.9). As a special case we get the following solution:

Corollary (7.10). For $S^{m} \times S^{n}=S^{6} \times S^{4}, S^{6} \times S^{5}, S^{8} \times S^{5}, S^{8} \times S^{6}, S^{9} \times S^{6}$, $S^{10} \times S^{6}$ one has an isomorphism of groups

$$
\mathcal{E}\left(q S^{m} \times S^{n}\right)=G_{q}(\pi, \pi, \varepsilon, \odot) .
$$

Here the right-hand side is the algebraically defined group in (7.8) depending only on the data in the list of (7.10). For example for $q S^{9} \times S^{6}$ we have $\varepsilon=-1$, $\pi=\mathbb{Z} / 24, \pi^{\prime}=\mathbb{Z} / 6, \pi_{1}=\mathbb{Z} / 2, \pi_{2}=(\mathbb{Z} / 2)^{3}$, and $\odot: \pi \otimes \pi=\mathbb{Z} / 2 \rightarrow \pi_{2}$ is the inclusion of the first summand $\mathbb{Z} / 2$ in $\pi_{2}$.

For a general connected sum $M$ of products of spheres we describe $\mathcal{E}(M)$, up to an extension problem, in terms of homotopy groups of spheres, see Theorem (7.6). The next result computes $\mathcal{E}(M)$ for a large class of highly connected manifolds completely. It can be applied to the connected sum $M=q S^{n} \times S^{n}$.

Theorem (8.14). Let $M$ be an $(n-1)$-connected $2 n$-dimensional manifold which is almost parallelizable, $n \geq 2$. Then one has the fundamental extension

$$
H_{n}(M) \otimes T_{n} \longmapsto \mathcal{E}(M) \rightarrow \operatorname{Aut}\left(\delta_{M}\right)
$$

where $\delta_{M}$ is the extended intersection form of $M$ and where $T_{n}=$ Tor $\Pi_{2 n+1}\left(S^{n+1}\right)$. This extension is split if in addition $n$ is odd or $n \in\{2,6\}$. For the canonical structure of $H_{n}(M) \otimes T_{n}$ as an Aut $\left(\delta_{M}\right)$-module, see (8.14).

In Theorem (8.14) we obtain a more general result for Poincaré-complexes. In the final section we compare diffeomorphisms of a manifold and homotopy equivalences; for almost parallelizable $(n-1)$-connected $2 n$-manifolds we describe the image of the canonical homomorphism

$$
\psi: \Pi_{0} \operatorname{Diff}(M) \rightarrow \mathcal{E}(M)
$$


in terms of the $\mathcal{J}$-homomorphism, see Theorem (10.3). For the Hopf-dimensions 4, 8 and 16 we get the following result where we do not assume that $M$ is almost parallelizable.

Theorem (9.3) and (9.5). Let $M$ be an $(n-1)$-connected $2 n$-dimensional manifold with $n \in\{2,4,8\}$. Then one has the fundamental extension

$$
\mathcal{E}(M \mid \dot{M}) \longmapsto \mathcal{E}(M) \rightarrow \operatorname{Aut}\left(\delta_{M}\right)
$$

which is split for $n=2$. Here $\mathcal{E}(M \mid \dot{M})$ is the following $\mathbb{Z} / 2$-vectorspace:

$$
\mathcal{E}(M \mid \dot{M}) \cong \begin{cases}\operatorname{Ker} \omega_{2}: H_{2}(M) \otimes \mathbb{Z} / 2 \rightarrow \mathbb{Z} / 2 & \text { for } n=2, \\ H_{4}(M) \otimes \mathbb{Z} / 2 & \text { for } n=4, \\ \left(H_{8}(M) \otimes \mathbb{Z} / 2\right)^{2} & \text { for } n=8, \omega \neq 0, \\ \left(H_{8}(M) \otimes \mathbb{Z} / 2\right)^{3} & \text { for } n=8, \omega=0 .\end{cases}
$$

The map $\omega_{2}$ is given by the second Stiefel-Whitney class of $M$ and we set $\omega \neq 0$ if the intersection form of $M$ is odd and $\omega=0$ otherwise. For the intricate structure of $\mathcal{E}(M \mid \dot{M})$ as an Aut $\left(\delta_{M}\right)$-module see $(9.5)$.

Hence this theorem determines $\mathcal{E}(M)$ for $n=2$ completely and for $n=4,8$ up to an extension problem. The case $n=2$ of the theorem is the main result in a recent paper of Cochran-Habegger [18]; the computation of $\mathcal{E}(M \mid \dot{M})$ for $n=2$ was already achieved in [11]. As a simple application we get, see (9.1):

$$
\mathcal{E}\left(\mathbb{C} P_{2}\right) \cong \mathbb{Z} / 2, \quad \mathcal{E}\left(\mathbb{H} P_{2}\right) \cong \mathbb{Z} / 2, \quad \mathcal{E}(\mathcal{C} a) \cong \mathbb{Z} / 2 \oplus \mathbb{Z} / 2 .
$$

The group $\mathcal{E}\left(\mathbb{R} P_{2}\right)$ is treated in [9]. The "quadratic algebra" developed in [13] is crucial for the proofs. Along the way we correct a collection of errors in the literature on the group $\mathcal{E}(M)$. The theorems in this introduction are applications and illustrations of our new techniques for the computation of $\mathcal{E}(M)$. We get the surprising general result:

Theorem (3.5). Let $M$ be an $(n-1)$-connected manifold of dimension $m \leq 3 n-2$, $n \geq 2$, and assume the attaching map for $M$ vanishes under suspension, i.e. $\Sigma f \simeq$ 0 . Then one has an isomorphism of $\mathcal{E}(\dot{M}, \pm f)$-modules

$$
\mathcal{E}(M \mid \dot{M}) \cong \operatorname{Im}\left\{\Sigma: \Pi_{m} \dot{M} \rightarrow \Pi_{m+1} \Sigma \dot{M}\right\}
$$

where $\Sigma$ is the suspension homomorphism.

We also compute $\mathcal{E}(M \mid \dot{M})$ for the delicate dimension $m=3 n-1$ and for connected sums $M=M_{0} \# M_{1}$. Moreover we obtain a criterion for the existence of a splitting of the fundamental extension, see $\S 5$. Further applications will appear in [15].

\section{The fundamental extension for $\mathcal{E}(M)$}

Let $n \geq 2$ and let $M$ be an $(n-1)$-connected closed manifold of dimension $m=2 n+k$ or more generally let $M$ be an $(n-1)$-connected Poincaré-complex of dimension $m=2 n+k, k \geq 0$. Poincaré-duality and homology decomposition [24] show that

$$
M=\dot{M} \cup_{f} e^{m}
$$


is obtained by attaching a cell $e^{m}$ where $\dot{M}$ has the homotopy type of a finite CW-complex with trivial $(n-1)$-skeleton and dimension $n+k$. For $k<n$ we see by the Freudenthal suspension theorem that $\dot{M}$ is homotopy equivalent to a suspension $\Sigma A$ where $A$ is a finite CW-complex with trivial $(n-2)$-skeleton and $\operatorname{dim}(A)=n+k-1$ (below we mainly deal with the case $k<n$ or equivalently $\operatorname{dim}(M)<3 n)$.

The coaction $\mu: M \rightarrow M \vee S^{m}$ is obtained by the contraction of the boundary of a small cell in $e^{m}$ to a point. This is the usual coaction for the mapping cone $C_{f}$ of the attaching map $f$ since by $(1.1)$ we have $M=C_{f}$. We denote by $[X, V]$ the set of homotopy classes of basepoint preserving maps $X \rightarrow V$ where we assume that $V$ is 1-connected and $X$ is well pointed, for example a manifold or a CW-complex. The coaction $\mu$ yields an action of the homotopy group $\Pi_{m}(M)$ on the homotopy set $[M, M]$,

$$
[M, M] \times \Pi_{m}(M) \stackrel{+}{\rightarrow}[M, M],
$$

which carries a pair $(x, \alpha)$ to $x+\alpha=(x, \alpha) \mu$. Clearly $\mathcal{E}(M)$ is the subgroup of units in the monoid $[M, M]$; the neutral element is given by the identity $1=1_{M}$. Let $\mathcal{E}(M \mid \dot{M})$ be the subgroup of $\mathcal{E}(M)$ consisting of all elements which can be represented by orientation preserving maps $M \rightarrow M$ which restrict to the identity of $\dot{M}$, or equivalently which are maps under $\dot{M}$. Moreover let $\mathcal{E}(\dot{M}, \pm f)$ be the group of all pairs $x=(x, \varepsilon) \in \mathcal{E}(\dot{M}) \times\{+1,-1\}$ for which $x_{*}: \Pi_{m-1} \dot{M} \rightarrow \Pi_{m-1} \dot{M}$ satisfies $x_{*} f=\varepsilon f$. Here $f \in \Pi_{m-1} \dot{M}$ is the homotopy class of the attaching map. We also write $\varepsilon=\operatorname{deg} x$; clearly $\operatorname{deg} x$ is determined by $x \in \mathcal{E}(\dot{M})$ if $2 f \neq 0$. The next result describes a crucial property of the group $\mathcal{E}(M)$; compare Barcus-Barratt [42] and Rutter [44].

(1.3) Proposition. For a 1-connected Poincaré-complex $M$ one has the short exact sequence of groups

$$
0 \rightarrow \mathcal{E}(M \mid \dot{M}) \rightarrow \mathcal{E}(M) \stackrel{r}{\rightarrow} \mathcal{E}(\dot{M}, \pm f) \rightarrow 0
$$

Here $\mathcal{E}(M \mid \dot{M})$ is an abelian group which is endowed with a surjective homomorphism of groups

$$
1^{+}: \Pi_{m}(\dot{M}) \rightarrow \mathcal{E}(M \mid \dot{M})
$$

defined by $1^{+}(\alpha)=1_{M}+i_{*} \alpha$. The map $i: \dot{M} \hookrightarrow M$ is the inclusion and + is given by the action (1.2). Moreover the structure of $\mathcal{E}(M \mid \dot{M})$ as a left $\mathcal{E}(\dot{M}, \pm f)$-module in the extension (a) can be described by the following formula where $a=1^{+}(\alpha) \in$ $\mathcal{E}(M \mid \dot{M})$ and $x \in \mathcal{E}(\dot{M}, \pm f)$,

$$
x \cdot a=1^{+}\left(\operatorname{deg}(x) \cdot x_{*}(\alpha)\right) .
$$

The fundamental extension (a) in (1.3) leads to three problems for the computation of $\mathcal{E}(M)$ : First one has to compute the group $\mathcal{E}(\dot{M}, \pm f)$, then one has to compute $\Pi_{m} \dot{M}$ and the kernel of $1^{+}$in (b), and finally one has to solve the extension problem for (a). 
(1.4) Theorem. For a 1-connected Poincaré-complex $M$ the abelian group $\mathcal{E}(M \mid \dot{M})$ is finite.

Proof. If $M$ is not rational equivalent to a sphere, this is proved by Aubry-Lemaire, see the Addendum in [4]. In case, however, $M$ is rational equivalent to a sphere, then $\dot{M}$ is a finite complex which is rational equivalent to a point and hence $\Pi_{m} \dot{M}$ is finite by a result of Sullivan. Hence then $\mathcal{E}(M \mid \dot{M})$ is also finite by (1.3).

\section{Poincaré-Complexes of Suspension-type}

We say that a manifold or a Poincaré-complex $M$ is of suspension-type if the space $\dot{M}$ in (1.1) has the homotopy type of a suspension, $\dot{M} \simeq \Sigma A$. For example a product $S^{n} \times S^{m}$ of two spheres is of suspension type. The next lemma yields many examples.

(2.1) Lemma. Let $M$ be an $(n-1)$-connected Poincaré-complex of dimension $m<$ $3 n$. Then $M$ is of suspension type. Moreover the connected sum $M=M_{0} \# M_{1}$ of manifolds $M_{0}, M_{1}$, which are both of suspension type, is again of suspension type.

By (1.3) we see that the group $\mathcal{E}(M \mid \dot{M})$ is a quotient of the homotopy group $\Pi_{m}(\dot{M})$. We now describe the relations for the quotient in case $M$ is of suspension type. To this end we need the Whitehead-product $[u, v] \in[\Sigma U \wedge V, X]$ for $u \in[\Sigma U, X], v \in[\Sigma V, X]$ and the James-Hopf-invariants $\gamma_{n} \beta \in\left[\Sigma U, \Sigma B^{\wedge n}\right]$ for $\beta \in[\Sigma U, \Sigma B]$. Here $B^{\wedge n}$ is the $n$-fold smash product $B \wedge \cdots \wedge B$ and the JamesHopf-invariant is defined with respect to the lexicographical ordering from the left, see [7]. Moreover we use for the one point union $U \vee V$ the partial suspension, $m \geq 2$,

$$
E: \Pi_{m-1}(U \vee V)_{2} \rightarrow \Pi_{m}(\Sigma U \vee V)_{2} .
$$

Here $\Pi_{k}(U \vee V)_{2}$ denotes the kernel of $r_{*}: \Pi_{k}(U \vee V) \rightarrow \Pi_{k}(V)$ where $r=$ $(0,1): U \vee V \rightarrow V$ is the retraction. Using the cone $C U$ of $U$ and the pinch map $\pi_{0}: C U \rightarrow C U / U=\Sigma U$ we obtain $E$ by the composition

$$
\begin{aligned}
& \Pi_{m-1}(U \vee V)_{2} \cong \Pi_{m}(C U \vee V, U \vee V) \\
& \downarrow\left(\pi_{0} \vee 1\right)_{*} \\
& \Pi_{m}(\Sigma U \vee V, V) \cong \Pi_{m}(\Sigma U \vee V)_{2} ;
\end{aligned}
$$

compare (II.11.8) of [8]. Let $i_{1}$, resp. $i_{2}$, be the inclusion of $U$, resp. $V$, into $U \vee V$. We define the difference operator $\nabla: \Pi_{m-1}(\Sigma A) \rightarrow \Pi_{m-1}(\Sigma A \vee \Sigma A)_{2}$ by $\nabla(f)=-f^{*}\left(i_{2}\right)+f^{*}\left(i_{2}+i_{1}\right)$.

The next theorem is based on 2.11 in [43].

(2.2) Theorem. Let $M$ be a 1-connected Poincaré complex of suspension type with $\dot{M}=\Sigma A$ and let $f: S^{m-1} \rightarrow \Sigma A$ be the attaching map. Then $1^{+}$in (1.3) (b) induces an isomorphism

$$
\mathcal{E}(M \mid \dot{M}) \cong \Pi_{m}(\Sigma A) / \mathcal{J} \quad \text { where } \mathcal{J}=\mathcal{J}_{M}=\operatorname{Im} \nabla(1, f)+\operatorname{Im} f_{*} .
$$

Here $f_{*}: \Pi_{m}\left(S^{m-1}\right) \rightarrow \Pi_{m}(\Sigma A)$ is induced by $f$ and $\nabla(1, f)$ is the homomorphism

$$
\nabla(1, f):\left[\Sigma^{2} A, \Sigma A\right] \rightarrow \Pi_{m}(\Sigma A)
$$


which is defined by the formula

$$
\begin{aligned}
\nabla(1, f)(\xi) & =(E \nabla f)^{*}(\xi, 1) \\
& =\xi \circ(\Sigma f)+[\xi, 1]\left(\Sigma \gamma_{2} f\right)+[[\xi, 1], 1]\left(\Sigma \gamma_{3} f\right)+\ldots
\end{aligned}
$$

Here $1=1_{\Sigma A}$ is the identity of $\Sigma A$ and the sum is taken over all summands $\omega_{n} \circ\left(\Sigma \gamma_{n} f\right), n \geq 1$, with $\omega_{1}=\xi$ and $\omega_{n}=\left[\omega_{n-1}, 1\right]$ for $n \geq 2$.

Clearly $\omega_{n} \circ\left(\Sigma \gamma_{n} f\right)$ is trivial if $n$ is sufficiently large since $\Sigma A$ is 1-connected.

We now describe an important property of Poincaré complexes of suspension type which was observed by Stöcker, see (6.1) [38]. For this we have to use SpanierWhitehead duality. For finite $\mathrm{CW}$-complexes $U, V$ let

$$
\{U, V\}=\lim \{[U, V] \stackrel{\Sigma}{\rightarrow}[\Sigma U, \Sigma V] \stackrel{\Sigma}{\rightarrow} \ldots\}
$$

be the group of stable maps $U \rightarrow V$. We have the stabilization $[U, V] \rightarrow\{U, V\}$. A map $u: S^{n} \rightarrow U \wedge V$ is a duality map if for any finite CW-complex $X$ the induced function

$$
u_{X}:\{U, X\} \rightarrow\left\{S^{n}, X \wedge V\right\}, \quad u_{X}\{f\}=\left\{f \wedge 1_{V}\right\}\{u\}
$$

is an isomorphism, see $(2,5,8)[36]$.

(2.4) Lemma [38]. Let $A$ be a connected finite $C W$-complex and let $f: S^{m-1} \rightarrow$ $\Sigma A$ be a map, $m \geq 4$. Then $M=\Sigma A \cup_{f} e^{m}$ is a Poincaré complex if and only if

$$
\Sigma \gamma_{2} f: S^{m} \rightarrow \Sigma^{2} A \wedge A=\Sigma A \wedge \Sigma A
$$

is a duality map.

The lemma characterizes all simply connected Poincaré complexes of suspension type. In particular for a Poincaré complex $M=\Sigma A \cup_{f} e^{m}$ the space $\Sigma A$ is self dual with respect to Spanier-Whitehead duality.

\section{3. $\Sigma$-REduciBle Poincaré-COMPleXes}

Following James [23] we shall say that a pointed space $Y$ is reducible if there is a map $f: S^{n} \rightarrow Y$ inducing isomorphisms of reduced homology groups $\widetilde{H}_{q}$ for all $q \geq n$. We say that $Y$ is $\Sigma^{r}$-reducible if the $r$-fold suspension $\Sigma^{r} Y$ is reducible.

(3.1) Remark. A finite complex $Y$ is $S$-reducible (stably reducible) if $Y$ is $\Sigma^{n}$ reducible for sufficiently large $n$, compare [3]. Using results of Atiyah [3] and Spivak [37] we have the following implications. A Poincaré-complex $M$ is $S$-reducible if and only if its Spivac-normal fibration is stably trivial. In particular a closed differentiable manifold $M$ is $S$-reducible if and only if its normal bundle or equivalently its tangent bundle is $\mathcal{J}$-trivial. This shows that an $S$-parallelizable manifold is also $S$-reducible.

We now consider $\Sigma$-reducible Poincaré-complexes.

(3.2) Lemma. Let $M=\dot{M} \cup_{f} e^{m}$ be a 1-connected Poincaré-complex. Then the following properties are equivalent:

(a) $M$ is $\Sigma$-reducible,

(b) there is a homotopy equivalence $\Sigma M \simeq \Sigma\left(S^{m} \vee \dot{M}\right)$,

(c) the suspension of $f$ is homotopically trivial, $\Sigma f \simeq 0$. 
Clearly a product $M=S^{n} \times S^{m}$ of spheres is $\Sigma$-reducible. Moreover the connected sum of $\Sigma$-reducible Poincaré complexes is again $\Sigma$-reducible. In addition we have the following examples.

(3.3) Lemma. Let $n \geq 3$ and let $M$ be an $(n-1)$-connected (2n)-manifold which is differential, or more generally for which $\dot{M}$ is differential. Then $M$ is $\Sigma$-reducible if $\dot{M}$ is stably parallelizable (or equivalently parallelizable). In particular $M$ is $\Sigma$ reducible if $n \equiv 3,5,6,7(8)$, i.e. $\Pi_{n-1} S O=0$.

The lemma follows from (8.10) (2) below; see the remark following the proof of $(8.22)$.

(3.4) Lemma. Let $M$ be a simply connected closed differential manifold of dimension $m=4$ or $m=5$. Then $M$ is $\Sigma$-reducible if and only if the second StiefelWhitney class $\omega_{2}$ vanishes. In particular each simply connected 5-dimensional Brieskorn manifold is $\Sigma$-reducible. See [15].

The next result computes the group $\mathcal{E}(M \mid \dot{M})$ of the fundamental extension for a large class of Poincaré complexes.

(3.5) Theorem. Let $M$ be an $(n-1)$-connected Poincaré complex of dimension $m \leq 3 n-2, n \geq 2$, and assume $M$ is $\Sigma$-reducible. Then one has the isomorphism of $\mathcal{E}(\dot{M}, \pm f)$-modules

$$
\mathcal{E}(M \mid \dot{M}) \cong \Sigma \Pi_{m}(\dot{M})
$$

where the right-hand side is the image of the suspension $\Sigma: \Pi_{m}(\dot{M}) \rightarrow \Pi_{m+1}(\Sigma \dot{M})$. More precisely one has $\operatorname{Ker} 1^{+}=\operatorname{Ker} \Sigma$ for $1^{+}$in (1.3).

This theorem is a consequence of the more general result (3.7) below. For this we use the EHP-sequence (in its extended form if $m=3 n-2$ ), see James [22], Baues [14](7.9).

For the delicate dimension $m=3 n-1$ we need the following condition $(*)$ which, in particular, is satisfied for 1-connected 5-dimensional Poincaré-complexes, see $[15]$.

(3.6) Definition. We say that $f: S^{m-1} \rightarrow \Sigma A$ satisfies condition $(*)$ if the equation

$$
\operatorname{Ker}[1,1]_{*}+\operatorname{Ker} \Sigma+\operatorname{Im} \overline{u_{\Sigma A}}=\Pi_{m} \Sigma A \wedge A
$$

holds. Here we use the homomorphisms $[1,1]_{*}: \Pi_{m} \Sigma A \wedge A \rightarrow \Pi_{m} \Sigma A, \Sigma: \Pi_{m} \Sigma A \wedge$ $A \rightarrow \Pi_{m+1} \Sigma^{2} A \wedge A, \bar{u}_{\Sigma A}:\left[\Sigma^{2} A, \Sigma A\right] \rightarrow \Pi_{m} \Sigma A \wedge A, \bar{u}_{\Sigma A}(\xi)=\left(\xi \wedge 1_{A}\right) \circ\left(\Sigma \gamma_{2} f\right)$.

(3.7) Theorem. Let $M$ be an $(n-1)$-connected Poincaré-complex of dimension $m=2 n+k<3 n, n \geq 2$. As in (2.1) we may assume that $M=\Sigma A \cup_{f} e^{m}$. If $M$ is $\Sigma$-reducible and if for $m=3 n-1$ condition $(*)$ is satisfied for $f$ then one has an isomorphism

$$
\mathcal{E}(M \mid \dot{M}) \cong \Pi_{m}(\Sigma A) / W .
$$

Here $W$ is the subgroup generated by all compositions $S^{m} \stackrel{\alpha}{\rightarrow} \Sigma A^{\wedge t} \stackrel{w^{t}}{\rightarrow} \Sigma A, 2 \leq t \leq$ 4 , where $w^{t}$ is any $t$-fold Whitehead-product of the identity $1_{\Sigma A}$. 
Proof. We consider the following commutative diagram where $X$ is an $(n-2)$ connected finite CW-complex and where $u$ is the stabilization of $\gamma_{2} f$, which is a duality map by (2.4).

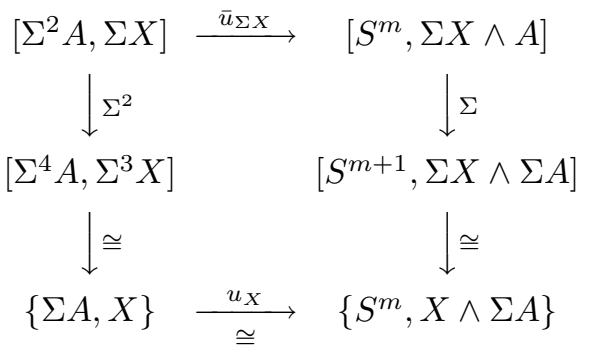

Here $u_{X}$ is defined as in (2.3) and $\bar{u}_{\Sigma X}$ carries an element $\xi \in\left[\Sigma^{2} A, \Sigma X\right]$ to $(\xi \wedge A)\left(\Sigma \gamma_{2} f\right)$ where $A$ denotes the identity $1_{A}$. The homomorphism $\Sigma$ in the diagram is surjective by the Freudenthal-suspension theorem. Moreover $\Sigma^{2}$ in (1) is surjective by the Freudenthal theorem for $X=A \wedge A$ and $X=A \wedge A \wedge A$. We also observe that $\Sigma$ in the diagram is an isomorphism for $n>2$; for $n=2$ the homomorphism $\Sigma$ is still an isomorphism for $X=A \wedge A$ or $X=A \wedge A \wedge A$. For $n=2$ and $X=A$ the kernel of $\Sigma$ is given by the image of

$$
\left[1_{\Sigma A \wedge A}, 1_{\Sigma A \wedge A}\right]_{*}: \Pi_{m} \Sigma A^{\wedge 4} \rightarrow \Pi_{m} \Sigma A^{\wedge 2} .
$$

Since we assume $\Sigma f=0$ we see by the extended EHP-sequence that we can write

$$
f=[1,1] \circ u+[[1,1], 1] \circ v .
$$

Here 1 is the identity of $\Sigma A$ and $u \in \Pi_{m-1}(\Sigma A \wedge A), v \in \Pi_{m-1}(\Sigma A \wedge A \wedge A)$. Only for $m=3 n-1$ the element $v$ might be nontrivial.

We have to show that the group $\mathcal{J}$ in (2.2) coincides with the group $W$ in the theorem. Since $\Sigma f=0$ it is easy to see by (3) and the definition of $\mathcal{J}$, that one has an inclusion $\mathcal{J} \subset W$. For this one only needs the Barcus-Barrat formula for Whitehead-products of the form $\left[\alpha \circ \beta, 1_{\Sigma A}\right]$. In our range this formula yields

$$
\left[\alpha \circ \beta, 1_{\Sigma A}\right]=\left[\alpha, 1_{\Sigma A}\right](\beta \wedge A)+\left[\left[\alpha, 1_{\Sigma A}\right], \alpha\right] T_{132}\left(\gamma_{2}(\beta) \wedge A\right)
$$

(compare [7]). For example for $[\xi, 1]$ in $(2.2)$ we set $\xi=\alpha \circ \beta$ with $\alpha=1_{\Sigma A}$ and $\beta=\xi$.

Next we show the inclusion $W \subset \operatorname{Im} \nabla(1, f)=\mathcal{J}^{\prime} \subset \mathcal{J}$. For this we first check that $w^{4} \circ \alpha \in \mathcal{J}^{\prime}$; see the definition of $w^{t}$ in (3.7) where we set $t=4$. By definition of $\nabla$ we know for any $\xi=[[1,1], 1] \circ \xi^{\prime}$ that

$$
\begin{aligned}
\nabla \xi & =\left[[[1,1], 1] \circ \xi^{\prime}, 1\right] \circ\left(\Sigma \gamma_{2} f\right) \\
& =[[[1,1], 1], 1]\left(\xi^{\prime} \wedge A\right) \circ\left(\Sigma \gamma_{2} f\right) \\
& =[[[1,1], 1], 1] \bar{u}_{\Sigma X}\left(\xi^{\prime}\right) \quad \text { with } X=A \wedge A \wedge A
\end{aligned}
$$

is an element in $\mathcal{J}^{\prime}$. On the other hand (1) shows that $\bar{u}_{\Sigma X}$ is surjective, hence also $w^{4} \circ \alpha \in \mathcal{J}^{\prime}$ for all four-fold Whitehead-products. At this point we also use the Jacobi-identity for Whitehead-products. 
Now we show, in a similar way, that all $w^{3} \circ \alpha$ are elements in $\mathcal{J}^{\prime}$. By definition of $\nabla$ we know for any $\xi=[1,1] \circ \xi^{\prime}$ that $\nabla \xi=(6)+(7)$,

$$
\begin{aligned}
& {\left[[1,1] \circ \xi^{\prime}, 1\right] \circ\left(\Sigma \gamma_{2} f\right),} \\
& {\left[\left[[1,1,] \circ \xi^{\prime}, 1\right], 1\right] \circ\left(\Sigma \gamma_{3} f\right),}
\end{aligned}
$$

is an element in $\mathcal{J}^{\prime}$. Here $(7)$ is of the form $w^{4} \circ \alpha$ which we have seen to be in $\mathcal{J}^{\prime}$ too. Therefore by (4) the element

$$
\begin{aligned}
(6) & =[[1,1], 1] \circ\left(\xi^{\prime} \wedge A\right) \circ\left(\Sigma \gamma_{2} f\right) \\
& =[[1,1], 1] \circ \bar{u}_{\Sigma X}\left(\xi^{\prime}\right) \quad \text { with } X=A \wedge A
\end{aligned}
$$

is in $\mathcal{J}^{\prime}$. Again (1) shows that here $\bar{u}_{\Sigma X}$ is surjective so that all elements $w^{3} \circ \alpha$ are in $\mathcal{J}^{\prime}$ where $w^{3}=[[1,1], 1]$.

Finally we show that all elements of the form $w^{2} \circ \alpha$ lie in $\mathcal{J}^{\prime}$. By definition of $\nabla$ we know for any $\xi$ that $\nabla \xi=(9)+(10)$,

$$
\begin{aligned}
& {[\xi, 1] \circ\left(\Sigma \gamma_{2} f\right),} \\
& {[[\xi, 1] 1] \circ\left(\Sigma \gamma_{3} f\right),}
\end{aligned}
$$

is an element in $\mathcal{J}^{\prime}$. Here we know that (10) is an element in $\mathcal{J}^{\prime}$ by the arguments above since we can use (4). On the other hand (4) shows $(9)=(11)+(12)$,

$$
\begin{aligned}
& {[1,1] \circ(\xi \wedge A) \circ\left(\Sigma \gamma_{2} f\right)=[1,1] \bar{u}_{\Sigma A}(\xi),} \\
& {[[1,1], 1] T_{132} \circ\left(\gamma_{2} \xi \wedge A\right) \circ\left(\Sigma \gamma_{2} f\right) .}
\end{aligned}
$$

Here we also know that (12) is an element in $\mathcal{J}^{\prime}$ so that therefore (11) is in $\mathcal{J}^{\prime}$ too. Using condition $(*)$ for $f$ diagram (1) and (2) show that for $X=A$ any element $\gamma \in\left[S^{m}, \Sigma A \wedge A\right]$ is of the form

$$
\gamma=\bar{u}_{\Sigma A}(\xi)+\left[1_{\Sigma A \wedge A}, 1_{\Sigma A \wedge A}\right] \circ \eta+\lambda
$$

where $\lambda \in \operatorname{Ker}[1,1]_{*}$. Therefore $[1,1] \circ \gamma=(11)+(14)$,

$$
[1,1] \circ\left[1_{\Sigma A \wedge A}, 1_{\Sigma A \wedge A}\right] \circ \eta=[[1,1],[1,1]] \circ \eta .
$$

The element (14) is of the form $w^{4} \circ \alpha$ and hence an element in $\mathcal{J}^{\prime}$. Therefore also $w^{2} \circ \gamma=[1,1] \circ \gamma$ is an element in $\mathcal{J}^{\prime}$ for all $\gamma$. This completes the proof that $W \subset \mathcal{J}^{\prime}$. This proof shows that actually $W=\mathcal{J}=\operatorname{Im} \nabla(1, f)$, see (2.2).

\section{Connected sums}

We consider the group $\mathcal{E}(M \mid \dot{M})$ in case $M=M_{0} \# M_{1}$ is a connected sum of $m$-dimensional Poincaré complexes. In this case the space $\dot{M}$ is the one point union $\dot{M}=\dot{M}_{0} \vee \dot{M}_{1}$. The attaching map $f$ of the $m$-cell in $M$ is the sum $f=i_{0} f-i_{1} f_{1}$ where $f_{\tau}$ is the attaching map in $M_{\tau}$ and where $i_{\tau}: M_{\tau} \subset M_{0} \vee M_{1}$ is the inclusion, $\tau \in\{0,1\}$. We can use

$$
M=M_{0} \# M_{1}=\left(\dot{M}_{0} \vee \dot{M}_{1}\right) \cup_{f} e^{m}
$$

as the definition of the connected sum of Poincaré complexes. This corresponds to the usual definition of the connected sum of manifolds. We derive from Theorem (3.5) immediately the following "additivity rule". 
(4.2) Theorem. Let $M_{0}$ and $M_{1}$ be $\Sigma$-reducible $(n-1)$-connected Poincaré complexes of dimension $m \leq 3 n-2$ and let $M=M_{0} \# M_{1}$ be the connected sum. Then one has the canonical isomorphism

$$
\mathcal{E}(M \mid \dot{M}) \cong \mathcal{E}\left(M_{0} \mid \dot{M}_{0}\right) \oplus \mathcal{E}\left(M_{1} \mid \dot{M}_{1}\right) .
$$

Proof. Using (3.5) we get $\mathcal{E}(M \mid \dot{M})=\Sigma \Pi_{m}\left(\dot{M}_{0} \vee \dot{M}_{1}\right)=\Sigma \Pi_{m} \dot{M}_{0} \oplus \Sigma \Pi_{m} \dot{M}_{1}=$ $\mathcal{E}\left(M_{0} \mid \dot{M}_{0}\right) \oplus \mathcal{E}\left(M_{1} \mid \dot{M}_{1}\right)$.

In general the additivity rule does not hold. For this we consider a connected sum $M=M_{0} \# M_{1}$ where $M_{0}$ is $\Sigma$-reducible and $M_{1}$ is not $\Sigma$-reducible.

(4.3) Theorem. Let $M_{0}$ and $M_{1}$ be $(n-1)$-connected Poincaré complexes of dimension $m<3 n-2$ and let $M=M_{0} \# M_{1}$ be the connected sum where $M_{0}$ is $\Sigma$-reducible. Then one has the isomorphism

$$
\mathcal{E}(M \mid \dot{M}) \cong \mathcal{E}\left(M_{0} \mid \dot{M}_{0}\right) / V \oplus \mathcal{E}\left(M_{1} \mid \dot{M}_{1}\right) .
$$

Here $V$ is the image of the homomorphism

$$
\left(\Sigma^{2} f_{1}\right)^{*}: \Sigma\left[\Sigma \dot{M}_{1}, \dot{M}_{0}\right] \rightarrow \Sigma \Pi_{m} \dot{M}_{0}=\mathcal{E}\left(M_{0} \mid \dot{M}_{0}\right)
$$

which carries an element $\Sigma \xi, \xi \in\left[\Sigma \dot{M}_{1}, \dot{M}_{0}\right]$, to the composition $(\Sigma \xi) \circ\left(\Sigma^{2} f_{1}\right)$ where $f_{1}$ is the attaching map of $M_{1}$.

We omit the proof since a more general result also for the delicate dimension $m=3 n-1$ is proved in [15].

\section{The extension Problem}

In the next two results we consider the extension problem in the fundamental extension for $\mathcal{E}(M)$ in (1.3). For this we consider the inclusion

$$
\Sigma \Pi_{m} \dot{M} \subset \Pi_{m+1} \Sigma \dot{M}
$$

as an inclusion of $\mathcal{E}(\dot{M}, \pm f)$-modules, the action of $x \in \mathcal{E}(\dot{M}, \pm f)$ on both sides being given by the formula $x \cdot a=\operatorname{deg}(x) \cdot(\Sigma x)_{*}(a)$.

(5.2) Theorem. Let $M$ be a 1-connected $\Sigma$-reducible Poincaré-complex and assume $\mathcal{E}(M \mid \dot{M}) \cong \Sigma \Pi_{m} \dot{M}$ as for example in (3.5). Moreover assume the inclusion (5.1) admits a retraction in the category of $\mathcal{E}(\dot{M}, \pm f)$-modules. Then the fundamental extension for $\mathcal{E}(M)$ is split.

Proof. We consider the following diagram in the category of groups

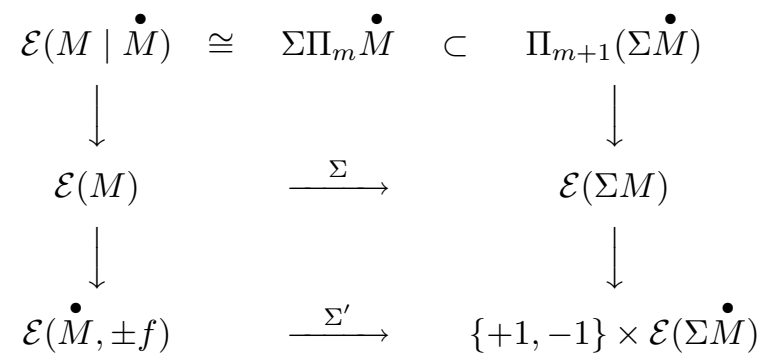


The assumptions in (5.2) imply that the upper square commutes. The lower square commutes since $\Sigma^{\prime}$ carries $x$ to the pair $(\operatorname{deg}(x), \Sigma x)$. Both columns are extension of groups. The right-hand side is split since $\Sigma M \simeq S^{m+1} \vee \Sigma \dot{M}$. Hence by the retraction for (5.1) also the left-hand side is split.

The next result can be applied for all $(n-1)$-Poincaré complexes of dimension $\leq 3 n-2$.

(5.3) Theorem. Let $M$ be a 1-connected Poincaré complex of suspension type with $\dot{M}=\Sigma A$ and let the attaching map of $M$ be of the form $f=[1,1] \circ u: S^{m-1} \rightarrow$ $\Sigma A \wedge A \rightarrow \Sigma A$. Moreover assume that $\Sigma:[A, A] \rightarrow[\Sigma A, \Sigma A]$ is injective and the composition

$$
\Pi_{m}(\Sigma A \wedge A) \stackrel{[1,1]^{*}}{\rightarrow} \Pi_{m}(\Sigma A) \stackrel{1^{+}}{\rightarrow} \mathcal{E}(M \mid \stackrel{\bullet}{M})
$$

is trivial. Then there is a homomorphism s for which the diagram

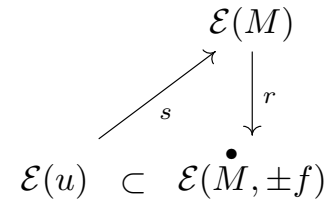

commutes. Here $\mathcal{E}(u)$ is the subgroup of $\mathcal{E}(\dot{M}, \pm f)$ consisting of all $\Sigma x \in \mathcal{E}(\Sigma A)$ with $x \in[A, A]$ and $(\Sigma x \wedge x)_{*} u= \pm u$.

Proof. For the proof we use the following explicit model $\omega_{A}$ of the Whitehead product $[1,1]$. The advantage of $\omega_{A}$ is its naturality, see (2) below. Let $C A$ be the reduced cone on $A$ and let $\pi_{0}: C A \rightarrow C A / A=\Sigma A$ be the quotient map. Then the product $\pi_{0} \times \pi_{0}$ yields the map of pairs

$$
\left.\pi_{0} \times \pi_{0}:(C A \times C A, C A \times C A) \rightarrow \Sigma A \times \Sigma A, \Sigma A \vee \Sigma A\right)
$$

where $C A \times C A=C A \times A \cup A \times C A$. Using the restriction of $\pi_{0} \times \pi_{0}$ we get the composition

$$
\omega_{A}: C A \dot{\times} C A \rightarrow \Sigma A \vee \Sigma A \stackrel{\nabla}{\rightarrow} \Sigma A
$$

where $\nabla$ is the folding map. It is clear that $\omega_{A}$ is natural with respect to pointed maps $\xi: A \rightarrow B$, that is

$$
(\Sigma \xi) \omega_{A}=\omega_{B} \hat{\xi}, \quad \hat{\xi}=C \xi \dot{\times} C \xi
$$

Since we assume that $A$ is well pointed we have a homotopy equivalence $h$ : $\Sigma(A \wedge A) \simeq C A \times C A$ and it is well known that $\omega_{A} h=[1,1]$ is the Whitehead product. We now define the section $s$ in (5.3) as follows. For $x \in \mathcal{E}(u)$ we choose a $\operatorname{map} \xi_{x}: A \rightarrow A$ in Top $^{*}$ such that $\Sigma \xi_{x}$ represents the homotopy class $x$. Moreover 
we consider the homotopy commutative diagram in Top*

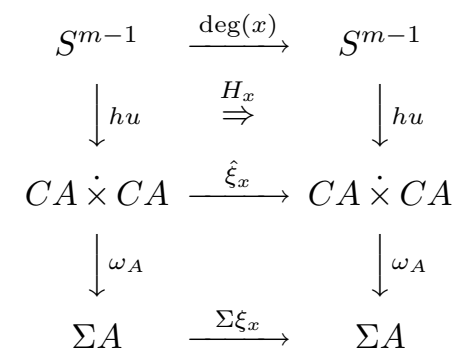

Here $H_{x}$ is a homotopy $\hat{\xi}_{x} h u \simeq(h u) \operatorname{deg}(x)$ which exists since $x \in \mathcal{E}(u)$. Moreover the bottom square commutes by (2). Hence we can define the principal map (with canonical $G$ )

$$
s(x)=C\left(\operatorname{deg}(x), \Sigma \xi_{x},\left(\omega_{A}\right)_{*} H_{x}, G\right) \in \mathcal{E}(M)
$$

(compare (V.2.2)[8]). Here $s(x)$ does not depend on the choice of $H_{x}$ since we assume $[1,1]_{*} 1^{+}=0$. It remains to show that $s$ is a homomorphism, that is, $s(x y)=(s x)(s y)$. Here $s(x), s(y)$ and $s(x y)$ are defined by the choices $\xi_{x}, \xi_{y}$ and $\xi_{x y}$ respectively. The composition $\xi_{x} \xi_{y}$ need not coincide with $\xi_{x y}$. The injectivity of $\Sigma$ in (5.3), however, implies that there is a homotopy $T: \xi_{x} \xi_{y} \simeq \xi_{x y}$. Now consider the diagrams as in (3) for $x, y$ and $x y$ respectively. These diagrams define an element $\alpha \in \Pi_{m}(\Sigma A)$ by the addition of tracks

$$
\alpha=\left(\Sigma \xi_{x}\right) \omega_{A} H_{y}+\omega_{A} H_{x} \operatorname{deg} y-\omega_{A} H_{x y}-(\Sigma T) \omega_{A} h u .
$$

Moreover we have $s(x y) \pm \alpha=(s x)(s y)$. Now (2) shows that $\alpha$ is of the form $\alpha=\omega_{A} \beta$ with

$$
\beta=\hat{\xi}_{x} H_{y}+H_{x} \operatorname{deg} y-H_{x y}-\widehat{T} h u .
$$

Hence $1^{+} \circ[1,1]_{*}=0$ implies $s(x y)=(s x)(s y)$.

\section{Products of SPheres}

We discuss the group of homotopy equivalences of a product of two spheres. We first consider the product $S^{n} \times S^{n}$. Let $\Delta_{8}$ be the subgroup of $G l_{2}(\mathbb{Z})$ generated by the matrices

$$
\left(\begin{array}{cc}
0 & -1 \\
1 & 0
\end{array}\right) \text { and }\left(\begin{array}{ll}
0 & 1 \\
1 & 0
\end{array}\right)
$$

This is the dihedral group of order eight (the group of symmetries of the square). Moreover let Sym be the infinite subgroup of $G l_{2}(\mathbb{Z})$ generated by

$$
\left(\begin{array}{ll}
0 & 1 \\
1 & 0
\end{array}\right), \quad\left(\begin{array}{ll}
1 & 2 \\
0 & 1
\end{array}\right) \text { and }\left(\begin{array}{cc}
0 & -1 \\
1 & 0
\end{array}\right) .
$$

Then we define the group $G_{n}, n \geq 1$, by

$$
G_{n}= \begin{cases}\Delta_{8}, & n \text { even, } \\ \text { Sym, } & n \text { odd, } n \neq 1,3,7, \\ G l_{2}(\mathbb{Z}), & n=1,3,7 .\end{cases}
$$


For a matrix $A=\left(\begin{array}{ll}a & b \\ c & d\end{array}\right) \in G_{n}$ we obtain $\operatorname{deg}(A) \in\{+1,-1\}$ by

$$
\operatorname{deg}(A)= \begin{cases}a d-b c & \text { if } n \text { is odd } \\ a d+b c & \text { if } n \text { is even }\end{cases}
$$

Now the fundamental extension for the group $\mathcal{E}\left(S^{n} \times S^{n}\right)$ is completely determined by the following result in which we use the torsion subgroup

$$
T_{n}=\operatorname{Tor} \Pi_{2 n+1}\left(S^{n+1}\right)=\Sigma \Pi_{2 n} S^{n}
$$

of the homotopy group $\Pi_{2 n+1}\left(S^{n+1}\right), n \geq 1$; for small values of $n$ we use the results of [39] to list $T_{n}$. (See Table 1.)

TABle 1

\begin{tabular}{|c||c|c|c|c|c|c|c|c|c|c|}
\hline$n$ & 1 & 2 & 3 & 4 & 5 & 6 & 7 & 8 & 9 & 10 \\
\hline$T_{n}$ & 0 & $\mathbb{Z}_{2}$ & $\mathbb{Z}_{12}$ & $\mathbb{Z}_{2}$ & 0 & $\mathbb{Z}_{2}$ & $\mathbb{Z}_{120}$ & $3 \mathbb{Z}_{2}$ & $3 \mathbb{Z}_{2}$ & $\mathbb{Z}_{12}$ \\
\hline
\end{tabular}

(6.3) Theorem. For $n \geq 1$ one has the split extension

$$
0 \rightarrow T_{n} \oplus T_{n} \rightarrow \mathcal{E}\left(S^{n} \times S^{n}\right) \rightarrow G_{n} \rightarrow 0 .
$$

Here $T_{n} \oplus T_{n}=(\mathbb{Z} \oplus \mathbb{Z}) \otimes T_{n}$ is a left $G_{n}$-module by $A \cdot(a \otimes t)=\operatorname{deg}(A) \cdot(A \cdot a) \otimes t$ for $A \in G_{n}, a \in \mathbb{Z} \oplus \mathbb{Z}, t \in T_{n}$.

Remark. The computation of $\mathcal{E}\left(S^{n} \times S^{n}\right)$ has an interesting history in the literature. The case $n=3$ was treated by Metzler-Zimmermann [33] and the case $n=7$ was considered by Sawashita [35]. The case $n=$ even was obtained in a paper [26], [27] by Kahn which, however, contains some odd "misprints". A complete solution as in (6.3) did not yet appear in the literature. For us Theorem (6.3) is an immediate consequence of (1.3), (3.5) and (5.2).

Proof of (6.3). For $n=$ odd, or $n=2$, or $n=6$ the result is a special case of (8.14) below. For $n=$ even one can define a splitting $s$ directly by the maps $f_{1}, f_{2}$ : $S^{n} \times S^{n} \rightarrow S^{n} \times S^{n}$ with $f_{1}(x, y)=(g(y), x)$ and $f_{2}(x, y)=(y, x)$; here $g: S^{n} \rightarrow S^{n}$ is a map of degree -1 . The splitting $s: \Delta_{8} \rightarrow \mathcal{E}\left(S^{n} \times S^{n}\right)$ is given by

$$
s\left(\begin{array}{cc}
0 & -1 \\
1 & 0
\end{array}\right)=f_{1}, \quad s\left(\begin{array}{ll}
0 & 1 \\
1 & 0
\end{array}\right)=f_{2} .
$$

Next we consider the group $\mathcal{E}\left(S^{m} \times S^{n}\right)$ with $2 \leq n<m$. Let $G_{m, n}$ be the set of all triples $(\mu, \nu, \xi)$ with $\mu, \nu \in\{+1,-1\}$ and $\xi \in \Pi_{m}\left(S^{n}\right)$ satisfying $\left[\iota_{n}, \xi\right]=0$ for the generator $\iota_{n} \in \Pi_{n} S^{n}$. The set $G_{m, n}$ is a group by the multiplication law:

$$
(\mu, \nu, \xi)\left(\mu^{\prime}, \nu^{\prime}, \xi^{\prime}\right)=\left(\mu \mu^{\prime}, \nu \nu^{\prime},\left(\nu \iota_{n}\right) \circ \xi^{\prime}+\mu^{\prime} \xi\right) .
$$

One readily checks that $G_{m, n}$ is a split extension

$$
0 \rightarrow \operatorname{Ker}\left[\iota_{n,-}\right] \rightarrow G_{m, n} \rightarrow\{+1,-1\} \times\{+1,-1\} \rightarrow 0 .
$$

The next result describes the fundamental extension for $\mathcal{E}\left(S^{m} \times S^{n}\right)$. 
(6.5) Theorem. For $2 \leq n<m$ one has the extension

$$
0 \rightarrow H_{m, n} \rightarrow \mathcal{E}\left(S^{m} \times S^{n}\right) \rightarrow G_{m, n} \rightarrow 0 .
$$

Here $H_{m, n}$ is given by the direct sum

$$
\begin{aligned}
H_{m, n} & =\Pi_{m+n}\left(S^{m}\right) /\left[\Pi_{n+1} S^{m}, \iota_{m}\right] \oplus \Pi_{m+n}\left(S^{n}\right) /\left[\Pi_{m+1} S^{n}, \iota_{n}\right] \\
& =\Sigma \Pi_{m+n}\left(S^{m}\right) \oplus \Sigma \Pi_{m+n}\left(S^{n}\right) \quad \text { for } m \leq 2 n-2 .
\end{aligned}
$$

Moreover $H_{m, n}$ is a left $G_{m, n}$-module by

$$
(\mu, \nu, \xi) \cdot(\{a\} \oplus\{b\})=\mu \nu\left(\left\{\left(\mu \iota_{m}\right) \circ a\right\} \oplus\left\{\left(\nu \iota_{n}\right) \circ b+\xi \circ a\right\}\right)
$$

where $a \in \Pi_{m+n}\left(S^{m}\right)$ and $b \in \Pi_{m+n}\left(S^{n}\right)$; the curly bracket denotes the corresponding coset (or the suspension for $m \leq 2 n-2)$.

Proof. For $M=S^{m} \times S^{n}$ the group $H_{m, n}=\mathcal{E}(M \mid \dot{M})$ was computed independently in [10] and [35], see also (7.6) below. Since the Whitehead product $f=\left[i_{1}, i_{2}\right]$ : $S^{m+n-1} \rightarrow S^{m} \vee S^{n}$ is the attaching map for $M$ one readily obtains the isomorphism $G_{m, n} \cong \mathcal{E}(\dot{M}, \pm f)$ which carries $(\mu, \nu, \xi)$ to the map $x: S^{m} \vee S^{n} \rightarrow S^{m} \vee S^{n}$ with $x i_{1}=\mu i_{1}+i_{2} \xi, x i_{2}=\nu i_{2}$. Here we get $\operatorname{deg}(x)=\mu \nu$. Hence the structure $H_{m, n}$ as a $G_{m, n}$-module is now obtained by (1.3) (c) and the left distributivity law.

In general the extension problem for $\mathcal{E}\left(S^{m} \times S^{n}\right)$ in $(6.5)$ is not solved. Sawashita obtained the remarkable result

(6.6) Theorem [35]. The fundamental extension for $\mathcal{E}\left(S^{3} \times S^{5}\right)$ is not split.

He also computes $\mathcal{E}\left(S^{m} \times S^{3}\right), m \geq 4$, and shows that this group is a split extension if $\omega_{3} \Sigma^{3}: \Pi_{n}\left(S^{3}\right) \rightarrow \Pi_{n+3}\left(S^{3}\right)$ is trivial. Here $\omega_{3} \in \Pi_{6}\left(S^{3}\right)$ is the generator of $\Pi_{6}\left(S^{3}\right)=\mathbb{Z}_{12}$ given by the commutator maps of the group $S^{3}$. Moreover he shows that the extension for $\mathcal{E}\left(S^{m} \times S^{2}\right), m \geq 3$, is always split. Also the extension for $\mathcal{E}\left(S^{n+1} \times S^{n}\right)$ is split for $n \not \equiv 3 \bmod 4$ and $n \neq 2,6$ since then $\operatorname{Ker}\left[\iota_{n,-}\right]=0$ in (6.4). We now use Theorem (5.2) to obtain many new cases for which the extension (6.5) is split.

(6.7) Theorem. Let $2 \leq n<m \leq 2 n-2$ and suppose that

$$
\Sigma: \Sigma \Pi_{n+m}\left(S^{n}\right) \rightarrow \Sigma \Pi_{n+m+1}\left(S^{n+1}\right)
$$

is injective and the image is a direct summand. Then the fundamental extension for $\mathcal{E}\left(S^{m} \times S^{n}\right)$ in (6.5) is split. This for example holds for $S^{6} \times S^{4}, S^{6} \times S^{5}$, $S^{8} \times S^{5}, S^{8} \times S^{6}, S^{9} \times S^{6}, S^{10} \times S^{6}$.

Proof. The assumptions imply that $\Sigma \Pi_{k}\left(S^{m}\right)=\Pi_{k+1}\left(S^{m+1}\right)$ with $k=n+m$. Hence the inclusion (5.1) for $M=S^{m} \times S^{n}$ is given by

$$
\Pi_{k+1}\left(S^{m+1}\right) \oplus \Sigma \Pi_{k}\left(S^{n}\right) \subset \Pi_{k+1}\left(S^{m+1}\right) \oplus \Pi_{k+1}\left(S^{n+1}\right) \oplus \mathbb{Z}
$$

where $\mathbb{Z}$ is generated by the Whitehead product $\left[i_{1}, i_{2}\right] \in \Pi_{k+1}\left(S^{m+1} \vee S^{n+1}\right)$. We now choose a retraction $R$ for $\Sigma$ in (6.7) and we define a retraction $r$ of (1) by

$$
r(a \oplus b \oplus t)=a \oplus R(\Sigma b)
$$

where $a \in \Pi_{k+1}\left(S^{m+1}\right), b \in \Pi_{k+1}\left(S^{n+1}\right), t \in \mathbb{Z}$. We claim that $r$ is actually a retraction of $\mathcal{E}(\dot{M}, \pm f)$-modules. For this we observe that $R$ satisfies $R\left(\Sigma^{2} b^{\prime}\right)=\Sigma b^{\prime}$ since $R$ is a retraction of $\Sigma$ in (6.7). For $(\mu, \nu, \xi) \in G_{m, n}$ we first get

$$
r(\mu, \nu, \xi)(a \oplus b \oplus t)=\mu \nu r\left(a^{\prime} \oplus b^{\prime} \oplus t^{\prime}\right)
$$


with

$$
a^{\prime}=\mu a, \quad b^{\prime}=\nu b+(\Sigma \xi) a+t\left[\Sigma \xi, \nu \iota_{n+1}\right], \quad t^{\prime}=\mu \nu t .
$$

Here we have (since $a=\Sigma a_{1}$ )

$$
R \Sigma b^{\prime}=R\left(\nu \Sigma b+\Sigma^{2} \xi \Sigma^{2} a_{1}\right)=R(\nu \Sigma b)+(\Sigma \xi) a
$$

so that by $(2)$

$$
(3)=\mu \nu(\mu a \oplus(R(\nu \Sigma b)+(\Sigma \xi) a)) .
$$

On the other hand we get

$$
\begin{aligned}
(\mu, \nu, \xi) \cdot r(a \oplus b \oplus t) & =(\mu, \nu, \xi)(a \oplus R \Sigma b) \\
& =\mu \nu(\mu a \oplus(\nu R \Sigma b+(\Sigma \xi) a))
\end{aligned}
$$

by the formula in (6.5). This shows that $(3)=(5)$ and hence we obtain the splitting for $\mathcal{E}\left(S^{m} \times S^{n}\right)$ by $(5.2)$.

\section{Connected sums of Products of Spheres}

We describe the fundamental extension for the group $\mathcal{E}(M)$ where $M$ is a connected sum of products of spheres. For this we need the following notation:

(7.1) Definition. We say that a 1-connected CW-space $X$ is spherical if (i) or equivalently (ii) is satisfied.

(i) There is an index set $R$ and a homotopy equivalence $X \simeq \bigvee_{r \in R} S_{r}$ where the right-hand side is a one point union of spheres $S_{r}=S^{n_{r}}, n_{r} \geq 2$.

(ii) The space $X$ has torsion free homology and the Hurewicz homomorphism $h: \Pi_{*} X \rightarrow \widetilde{H}_{*} X$ is surjective.

For the graded homotopy group $\Pi_{*} X$ of a spherical space $X$ we define the graded submodule

$$
\left[\Pi_{*} X, \widetilde{H}_{*} X\right] \subset \Pi_{*} X
$$

as follows. Let $s: \widetilde{H}_{*} X \rightarrow \Pi_{*} X$ be any degree 0 homomorphism with $h s=$ id. Clearly $s$ exists by (7.1) (ii). Then $\left[\Pi_{*} X, \widetilde{H}_{*} X\right]_{m} \subset \Pi_{m} X$ is generated by all Whitehead products $[\alpha, s \beta]$ with $\alpha \in \Pi_{*} X, \beta \in \widetilde{H}_{*} X$ and $|\alpha|+|\beta|-1=m$. Choosing the splitting $s$ of $h$ by the equivalence in (7.1) (i) we get

$$
\left[\Pi_{*} X, \widetilde{H}_{*} X\right]_{m}=\sum_{r \in R}\left[\Pi_{m-n_{r}+1}(X), i_{r}\right]
$$

where $i_{r}: S^{n_{r}} \rightarrow X$ is given by the one point union in (7.1) (i).

(7.4) Lemma. For a spherical space $X$ the submodule $\left[\Pi_{*} X, \widetilde{H}_{*} X\right]$ of $\Pi_{*} X$ does not depend on the choice of the splitting s above. Moreover $\left[\Pi_{*} X, \widetilde{H}_{*} X\right]$ is an $\mathcal{E}(X)$-submodule of the $\mathcal{E}(X)$-module $\Pi_{*} X$.

The lemma can be proved by the Hilton-Milnor theorem. We now consider a connected sum

$$
M=\left(S^{a_{1}} \times S^{b_{1}}\right) \#\left(S^{a_{2}} \times S^{b_{2}}\right) \# \cdots \#\left(S^{a_{q}} \times S^{b_{q}}\right)
$$

of products of spheres with $q \geq 1, a_{r}+b_{r}=m, 2 \leq b_{r} \leq a_{r}, r \in\{1, \ldots, q\}$. Clearly $\dot{M}$ is spherical. 
(7.6) Theorem. For a connected sum $M$ of products of spheres as above the group $\mathcal{E}(\dot{M}, \pm f)$ is completely determined by homotopy groups of spheres. Moreover one has an isomorphism

$$
\mathcal{E}(M \mid \dot{M}) \cong \Pi_{m}(\dot{M}) /\left[\Pi_{*} \dot{M}, \widetilde{H}_{*} \dot{M}\right]_{m}
$$

of $\mathcal{E}(\dot{M}, \pm f)$-modules where the right-hand side is again completely determined by the homotopy groups of spheres, see (7.2). For example if $M$ is $(n-1)$-connected and $\operatorname{dim} M \leq 3 n-2$ we get

$$
\mathcal{E}(M \mid \dot{M}) \cong \bigoplus_{k} \widetilde{H}_{k}(\dot{M}) \otimes \Sigma \Pi_{m}\left(S^{k}\right) .
$$

The theorem shows that the group $\mathcal{E}(M)$ is computable up to an extension problem once one has enough knowledge of the structure of homotopy groups of spheres. Clearly Theorem (7.6) yields Theorem (6.5) as a special case.

Proof. The Hilton-Milnor theorem shows that $\mathcal{E}(\dot{M}, \pm f)$ and $\Pi_{m}(\dot{M})$ are determined by homotopy groups of spheres. The composition in $\mathcal{E}(\dot{M}, \pm f)$ and the Whitehead product in $\Pi_{*}(\dot{M})$ involve the distributivity laws of homotopy theory. For this suspension, Hopf invariants and Whitehead products are needed which we consider as a part of the structure of homotopy groups of spheres. We now show that the first isomorphism for $\mathcal{E}(M \mid \dot{M})$ in (7.6) holds. For this we use Theorem (2.2); the second isomorphism in (7.6) is an immediate consequence of (3.5). We have

$$
\begin{aligned}
\dot{M} & =\Sigma A \vee \Sigma B \quad \text { where } \\
\Sigma A & =S^{a_{1}} \vee \cdots \vee S^{a_{q}}, \\
\Sigma B & =S^{b_{1}} \vee \cdots \vee S^{b_{q}} .
\end{aligned}
$$

Moreover the attaching map $f$ for $M$ can be chosen to be the sum of Whitehead products

$$
f=\sum_{r=1}^{q}\left[\alpha_{r}, \beta_{r}\right] \in \Pi_{m-1}(\dot{M})
$$

where $\alpha_{r}: S^{a_{1}} \subset \Sigma A \subset \dot{M}$ and $\beta_{r}: S^{b_{r}} \subset \Sigma B \subset \dot{M}$ are the inclusions. For $A_{1}=A_{2}=A$ and $B_{1}=B_{2}=B$ we get the difference element

$$
\begin{aligned}
\nabla f & : S^{m-1} \rightarrow \Sigma A_{1} \vee \Sigma B_{1} \vee \Sigma A_{2} \vee \Sigma B_{2}, \\
\nabla f & =-i_{2} f+\left(i_{2}+i_{1}\right) f \\
& =\sum_{r=1}^{q}\left(-\left[i_{A}^{2} \alpha_{r}, i_{B}^{2} \beta_{r}\right]+\left[\left(i_{A}^{2}+i_{A}^{1}\right) \alpha_{r},\left(i_{B}^{2}+i_{B}^{1}\right) \beta_{r}\right]\right) \\
& =\sum_{r=1}^{q}\left(\left[i_{A}^{1} \alpha_{r}, i_{B}^{2} \beta_{r}\right] \pm\left[i_{B}^{2} \beta_{r}, i_{A}^{1} \alpha_{r}\right]+\left[i_{A}^{1} \alpha_{r}, i_{B}^{1} \beta_{r}\right]\right) .
\end{aligned}
$$

Here $i_{A}^{\tau}, i_{B}^{\tau}, \tau \in\{1,2\}$, denote the corresponding inclusions of $\Sigma A$ and $\Sigma B$ respectively. Now the rules for the partial suspension $E$ show $E\left[i_{A}^{1} \alpha_{r}, i_{B}^{1}, \beta_{r}\right]=0$ 
and

$$
E \nabla f=\sum_{r=1}^{q}\left(\left[\Sigma i_{A}^{1} \alpha_{r}, i_{B}^{2} \beta_{r}\right] \pm\left[\Sigma i_{B}^{2} \beta_{r}, i_{A}^{1} \alpha_{r}\right]\right) .
$$

For $\xi=\left(\xi_{A}, \xi_{B}\right) \in\left[\Sigma^{2} A \vee \Sigma^{2} B, \Sigma A \vee \Sigma B\right]$ we thus get

$$
\begin{aligned}
\nabla(1, f)(\xi) & =(E \nabla f)^{*}(\xi, 1) \\
& =\sum_{r=1}^{q}\left(\left[\xi_{A}\left(\Sigma \alpha_{r}\right), i_{B} \beta_{r}\right] \pm\left[\xi_{B}\left(\Sigma \beta_{r}\right), i_{A} \alpha_{r}\right]\right) .
\end{aligned}
$$

This implies by (7.3)

$$
\operatorname{Im} \nabla(1, f)=\left[\Pi_{*} \dot{M}, \widetilde{H}_{*} \dot{M}\right]_{m} .
$$

Moreover $\operatorname{Im} f_{*} \subset \operatorname{Im} \nabla(1, f)$ since for the generator $\eta \in \Pi_{m} S^{m-1}=\mathbb{Z} / 2$

$$
f \circ \eta=\sum_{r}\left[\alpha_{r}, \beta_{r}\right] \eta=\sum_{r}\left[\alpha_{r} \eta, \beta_{r}\right] \in\left[\Pi_{*} \dot{M}, \widetilde{H}_{*} \dot{M}\right] .
$$

This completes by (5.2) the proof of (7.6).

As an illustration of (7.6) we consider the example of a $q$-fold connected sum

$$
q S^{m} \times S^{n}=\left(S^{m} \times S^{n}\right) \# \cdots \#\left(S^{m} \times S^{n}\right) .
$$

The case $m=n$ is treated in the next section ( $(8)$ so that we assume $n \neq m$. We need the following types of algebraic groups.

(7.8) Definition. Let $G l_{q}(\mathbb{Z})$ be the general linear group, the automorphism group of $\mathbb{Z}^{q}$. For an abelian group $\Pi$ let $\Pi^{q}=\Pi \oplus \cdots \oplus \Pi$ be the $q$-fold direct sum and let $M_{q}(\Pi)$ be the abelian group of $q \times q$-matrices $\xi=\left(\xi_{s}^{r}\right)$ with entries $\xi_{s}^{r} \in \Pi$. Then $M_{q}(\Pi)$ and $\Pi^{q}$ are left and right $G l_{q}(\mathbb{Z})$-modules in the usual way. For a subgroup $\Pi^{\prime}$ of $\Pi$ and for $\varepsilon \in\{+1,-1\}$ with $(1+\varepsilon) \Pi \subset \Pi^{\prime}$ let

$$
M_{q}\left(\Pi, \Pi^{\prime}, \varepsilon\right) \subset M_{q}(\Pi)
$$

be the subgroup consisting of all matrices $\xi$ with $\xi_{r}^{r} \in \Pi^{\prime}$ and $\xi_{s}^{r}=\varepsilon \xi_{r}^{s}$ for $r \neq s$. We now define a group $G_{q}\left(\Pi, \Pi^{\prime}, \varepsilon\right)$ which as a set is the product

$$
G_{q}\left(\Pi, \Pi^{\prime}, \varepsilon\right)=M_{q}\left(\Pi, \Pi^{\prime}, \varepsilon\right) \times G l_{q}(\mathbb{Z}) \times\{+1,-1\} .
$$

The group structure is given by

$$
(\xi, N, \delta) \cdot\left(\xi^{\prime}, M, \delta^{\prime}\right)=\left(\delta^{\prime} M^{-1} \xi\left({ }^{t} M\right)^{-1}+\xi^{\prime}, N M, \delta \delta^{\prime}\right) .
$$

Here ${ }^{t} M$ is the transpose of $M \in G l_{q}(\mathbb{Z})$. Clearly one has the split extension

$$
M_{q}\left(\Pi, \Pi^{\prime}, \varepsilon\right) \stackrel{i}{\longmapsto} G_{q}\left(\Pi, \Pi^{\prime}, \varepsilon\right) \stackrel{r}{\rightarrow} G l_{q}(\mathbb{Z}) \times\{+1,-1\}
$$

with $r(\xi, N, \delta)=(N, \delta), i(\xi)=(\xi, 1,+1)$. Here $1 \in G l_{q}(\mathbb{Z})$ is the neutral element. The splitting $\sigma$ of $r$ is $\sigma(N, \delta)=(0, N, \delta)$. Next let $\Pi_{1}$ and $\Pi_{2}$ be abelian groups and let

$$
\odot: \Pi \otimes \Pi_{1} \rightarrow \Pi_{2}, \quad a \otimes \lambda \mapsto a \odot \lambda
$$

be a homomorphism. Then the direct sum $\Pi_{1}^{q} \oplus \Pi_{2}^{q}$ is a $G_{q}\left(\Pi, \Pi^{\prime}, \varepsilon\right)$-module by the action

$$
(\xi, N, \delta) \cdot(a \oplus b)=\left({ }^{t} N^{-1} \cdot a\right) \oplus \delta N(\xi \odot a+b)
$$


for $a \in \Pi_{1}^{q}, b \in \Pi_{2}^{q}$. Here (4) yields the canonical extension

$$
\odot: M_{q}(\Pi) \otimes \Pi_{1}^{q} \rightarrow \Pi_{2}^{q},
$$

which carries $\xi \otimes a$ to $\xi \odot a$, with coordinates

$$
(\xi \odot a)_{s}=\sum_{r=1}^{q} \xi_{s}^{r} \odot a_{r}
$$

for $a=a_{1} \oplus \cdots \oplus a_{q} \in \Pi_{1}^{q}$. The action (5) defines the group $G_{q}\left(\Pi, \Pi^{\prime}, \varepsilon, \odot\right)$ which is the split extension

$$
\Pi_{1}^{q} \oplus \Pi_{2}^{q} \longmapsto G_{q}\left(\Pi, \Pi^{\prime}, \varepsilon, \odot\right) \rightarrow G_{q}\left(\Pi, \Pi^{\prime}, \varepsilon\right) .
$$

Using these algebraic groups we are now ready to describe the fundamental extension for the $q$-fold connected sum $q S^{m} \times S^{n}$.

(7.9) Theorem. Let $2 \leq n<m \leq 2 n-2$ and $q \geq 1$. Then one has the extension of groups

$$
\Pi_{1}^{q} \oplus \Pi_{2}^{q} \longmapsto \mathcal{E}\left(q S^{m} \times S^{n}\right) \stackrel{r}{\rightarrow} G_{q}\left(\Pi, \Pi^{\prime}, \varepsilon\right)
$$

where

$$
\begin{aligned}
\Pi_{1} & =\Sigma \Pi_{m+n}\left(S^{m}\right), \quad \Pi_{2}=\Sigma \Pi_{m+n}\left(S^{n}\right), \\
\varepsilon & =(-1)^{n-1}, \quad \Pi=\Pi_{m} S^{n}, \\
\Pi^{\prime} & =\operatorname{Ker}\left\{\left[i_{n}, i_{n}\right]_{*} \Sigma^{n-1}: \Pi_{m} S^{n} \rightarrow \Pi_{m+n-1} S^{n}\right\} .
\end{aligned}
$$

The $G_{q}\left(\Pi . \Pi^{\prime}, \varepsilon\right)$-module $\Pi_{1}^{q} \oplus \Pi_{2}^{q}$ in (1) is determined as in (7.8) (5) by the homomorphism

$$
\odot: \Pi_{m} S^{n} \otimes \Sigma \Pi_{m+n} S^{m} \rightarrow \Sigma \Pi_{m+n} S^{n}
$$

which carries $\xi \otimes \Sigma \alpha$ to the composition $\Sigma(\xi \circ \alpha)$. The extension (1) admits a partial splitting s for which the diagram

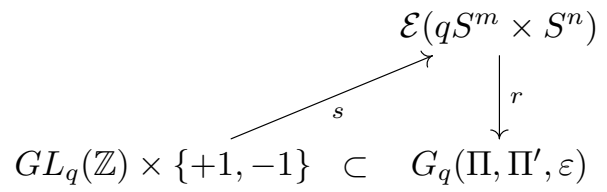

commutes. Moreover the extension (1) is split if in addition $\Sigma: \Sigma \Pi_{m+n}\left(S^{n}\right) \rightarrow$ $\Sigma \Pi_{m+n+1}\left(S^{n+1}\right)$ is injective and a direct summand; hence in this case we have the isomorphism of groups

$$
\mathcal{E}\left(q S^{m} \times S^{n}\right) \cong G_{q}\left(\Pi, \Pi^{\prime}, \varepsilon, \odot\right) .
$$

The next corollary shows explicit examples for which the computation of the group $\mathcal{E}\left(q S^{M} \times S^{n}\right)$ is achieved completely.

(7.10) Corollary. For $S^{m} \times S^{n}=S^{6} \times S^{4}, S^{6} \times S^{5}, S^{8} \times S^{5}, S^{8} \times S^{6}, S^{9} \times S^{6}$, $S^{10} \times S^{6}$ we have an isomorphism of groups

$$
\mathcal{E}\left(q S^{m} \times S^{n}\right)=G_{q}\left(\Pi, \Pi^{\prime}, \varepsilon, \odot\right)
$$

where $\Pi, \Pi^{\prime}, \varepsilon, \odot$ are given in Table 2. 
TABLE 2

\begin{tabular}{|c||l|l|l|l|l|l|}
\hline$S^{m} \times S^{n}$ & $\varepsilon$ & $\Pi$ & $\Pi^{\prime}$ & $\Pi_{1}$ & $\Pi_{2}$ & $\odot$ \\
\hline \hline$S^{6} \times S^{4}$ & -1 & $\mathbb{Z}_{2}$ & 0 & 0 & $\mathbb{Z}_{2}$ & 0 \\
\hline$S^{6} \times S^{5}$ & +1 & $\mathbb{Z}_{2}$ & 0 & 0 & $\mathbb{Z}_{2}$ & 0 \\
\hline$S^{8} \times S^{5}$ & +1 & $\mathbb{Z}_{24}$ & $\mathbb{Z}_{24}$ & 0 & $\mathbb{Z}_{2}$ & 0 \\
\hline$S^{8} \times S^{6}$ & -1 & $\mathbb{Z}_{2}$ & $\mathbb{Z}_{2}$ & $\mathbb{Z}_{2}$ & $\left(\mathbb{Z}_{2}\right)^{2}$ & 0 \\
\hline$S^{9} \times S^{6}$ & -1 & $\mathbb{Z}_{24}$ & $\mathbb{Z}_{6}$ & $\mathbb{Z}_{2}$ & $\left(\mathbb{Z}_{2}\right)^{3}$ & $\neq 0$ \\
\hline$S^{10} \times S^{6}$ & -1 & 0 & 0 & $\mathbb{Z}_{2}$ & $\mathbb{Z}_{2} \oplus \mathbb{Z}_{24}$ & 0 \\
\hline
\end{tabular}

The list is obtained by an elaborate inspection of Toda's book [39]. For $S^{9} \times S^{6}$ we obtain $\odot \neq 0$ since $\nu_{6} \in \Pi, \nu_{9} \nu_{12} \in \Pi_{1}$ and $0 \neq \nu_{6} \nu_{9} \nu_{12} \in \Pi_{2}$. For $S^{8} \times S^{6}$ we get $\odot=0$ since $\eta_{6} \eta_{7} \in \Pi, \nu_{8} \nu_{11} \in \Pi_{1}$ and $\eta_{6} \eta_{7} \nu_{8} \nu_{11}=\eta_{6} \Sigma^{4}\left(\nu^{\prime} \eta_{6}\right) \nu_{11}=\eta_{6}\left(\Sigma^{4} \nu^{\prime}\right) \eta_{10} \nu_{11}$ where $\eta_{6}\left(\Sigma^{4} \nu^{\prime}\right) \eta_{10}=0$.

Proof of (7.9). We use the same notation as in the proof of (7.6). Hence the attaching map for $M=q S^{m} \times S^{n}$ is

$$
\begin{cases}f: S^{m+n-1} \rightarrow \Sigma A \vee \Sigma B, & \Sigma A=S^{m} \vee \cdots \vee S^{m}, \\ f=\Sigma_{r=1}^{q}\left[\alpha_{r}, \beta_{r}\right], & \Sigma B=S^{n} \vee \cdots \vee S^{n},\end{cases}
$$

where $\alpha_{r}$ and $\beta_{r}$ are the inclusions of $S^{m}$ and $S^{n}$ respectively. We identify

$$
\left\{\begin{array}{l}
G l_{q}(\mathbb{Z})=\mathcal{E}(\Sigma A)=\mathcal{E}(\Sigma B), \\
M_{q}(\Pi)=[\Sigma A, \Sigma B],
\end{array}\right.
$$

where we use the assumption $m \leq 2 n-2$. For a map

$$
(L+\eta, N): \Sigma A \vee \Sigma B \rightarrow \Sigma A \vee \Sigma B
$$

with $L \in \mathcal{E}(\Sigma A), N \in \mathcal{E}(\Sigma B), \eta \in[\Sigma A, \Sigma B]$ we have $(L+\eta, N) \in \mathcal{E}(\dot{M}, \pm f)$ if and only if the following equation holds, $\delta \in\{+1,-1\}$ :

$$
\sum_{r=1}^{q}\left[(L+\eta) \alpha_{r}, N \beta_{r}\right]=\delta \sum_{r=1}^{q}\left[\alpha_{r}, \beta_{r}\right] .
$$

This is equivalent to (4) and (5)

$$
\begin{aligned}
& \sum_{r=1}^{q}\left[L \alpha_{r}, N \beta_{r}\right]=\delta \sum_{j=1}^{q}\left[\alpha_{j}, \beta_{j}\right], \\
& \sum_{r=1}^{q}\left[\eta \alpha_{r}, N \beta_{r}\right]=0 .
\end{aligned}
$$

For $L \alpha_{r}=\sum_{s} L_{s}^{r} \alpha_{s}$ and $N \beta_{r}=\sum_{t} N_{t}^{r} \beta_{t}$ we thus get equivalently to (4) the equations

$$
\sum_{r} L_{j}^{r} N_{j}^{r}=\delta \quad \text { and } \sum_{r} L_{s}^{r} N_{t}^{r}=0 \quad \text { for } s \neq t
$$

or equivalently

$$
L=\delta^{t} N^{-1}
$$


Next let $\xi=N^{-1} \cdot \eta$. Then we have $\xi \alpha_{r}=\sum_{s} \beta_{s} \xi_{s}^{r}$ and hence (5) is equivalent to

$$
0=\sum_{r=1}^{q}\left[\xi \alpha_{r}, \beta_{r}\right]=\sum_{r, s}\left[\beta_{s} \xi_{s}^{r}, \beta_{r}\right]
$$

Here we have

$$
\left[\beta_{r} \xi_{r}^{r}, \beta_{r}\right]=\beta_{r}\left[i_{n}, i_{n}\right]\left(\xi_{r}^{r} \wedge S^{n-1}\right)
$$

and for $r<s$

$$
\begin{aligned}
{\left[\beta_{s} \xi_{s}^{r}, \beta_{r}\right]+\left[\beta_{r} \xi_{r}^{s}, \beta_{s}\right] } & =\left[\beta_{s}, \beta_{r}\right] \xi_{s}^{r} \wedge S^{n-1}+\left[\beta_{r}, \beta_{s}\right] \xi_{r}^{s} \wedge S^{n-1} \\
& =\left[\beta_{s}, \beta_{r}\right]\left(\xi_{s}^{r} \wedge S^{n-1}-(-1)^{n-1} \xi_{r}^{s} \wedge S^{n-1}\right) .
\end{aligned}
$$

Here $\alpha \wedge S^{n-1}=\chi_{n, m} \Sigma^{n-1} \alpha$ with $\chi_{n, m} \in\{+1,-1\}$ for all $\alpha \in \Pi_{m} S^{n}$. Now (9) and (10) show that (8) is equivalent to $\xi \in M_{q}\left(\Pi, \Pi^{\prime}, \varepsilon\right)$. Hence we get the isomorphism

$$
G_{q}\left(\Pi, \Pi^{\prime}, \varepsilon\right) \cong \mathcal{E}(\dot{M}, \pm f)
$$

which carries $(\xi, N, \delta)$ to $\left(\delta^{t} N^{-1}+N \xi, N\right)$. The multiplication law in $\mathcal{E}(\dot{M}, \pm f)$ satisfies

$$
(L+\eta, N)\left(L^{\prime}+\eta^{\prime}, N^{\prime}\right)=\left(L L^{\prime}+\eta L^{\prime}+N \eta^{\prime}, N N^{\prime}\right)
$$

which shows that (11) is an isomorphism of groups, see (7.8) (2). Using the isomorphism (11) we also get by (1.3) (c) the formula for the action in (7.8) (5). Now the proof of the first part of (7.9) is complete by (3.5). We obtain the splitting $s$ in (7.9) (2) by (5.3) and (7). Finally we get the isomorphism (7.9) (3) by (5.2) similarly as in (6.7).

\section{8. $(n-1)$-CONNECTED $2 n$-MANIFOLDS}

We first introduce the algebra which is needed for the metastable range of homotopy theory; for a more extensive treatment see [13].

(8.1) Definition. A quadratic $\mathbb{Z}$-module

$$
M=\left(M_{e} \stackrel{H}{\rightarrow} M_{e e} \stackrel{P}{\rightarrow} M_{e}\right)
$$

is a pair of abelian groups $M_{e}, M_{e e}$ together with homomorphisms $H, P$ which satisfy

$$
P H P=2 P \quad \text { and } \quad H P H=2 H .
$$

Then $T=H P-1$ is an involution on $M_{e e}$, i.e. $T T=1$. A morphism $f: M \rightarrow N$ between quadratic $\mathbb{Z}$-modules is a pair of homomorphisms $f=\left(f_{e}, f_{e e}\right)$ which commute with $H$ and $P$ respectively, $f_{e} P=P f_{e e}, f_{e e} H=H f_{e}$. Let $\mathbf{Q M}(\mathbb{Z})$ be the category of quadratic $\mathbb{Z}$-modules which is an abelian category. We identify an abelian group $\Pi$ with the quadratic $\mathbb{Z}$-module $\Pi=(\Pi \rightarrow 0 \rightarrow \Pi)$; this yields the inclusion $\mathbf{M}(\mathbb{Z}) \subset \mathbf{Q M}(\mathbb{Z})$ where $\mathbf{M}(\mathbb{Z})=\mathbf{A b}$ is the category of abelian groups (i.e. $\mathbb{Z}$-modules).

(8.2) Definition. Let $A$ be an abelian group and let $M$ be a quadratic $\mathbb{Z}$-module. A quadratic form $A \rightarrow M$ is given by a pair of functions $\alpha=\left(\alpha_{e}, \alpha_{e e}\right)$ where $\alpha_{e e}: A \times A \rightarrow M_{e e}$ is $\mathbb{Z}$-bilinear and where $\alpha_{e}: A \rightarrow M_{e}$ satisfies for $a, b \in A$

$$
\left\{\begin{array}{l}
\alpha_{e}(a+b)=\alpha_{e}(a)+\alpha_{e}(b)+P \alpha_{e e}(a, b), \\
\alpha_{e e}(a, a)=H \alpha_{e}(a) .
\end{array}\right.
$$


Let $\operatorname{Hom}_{\mathbb{Z}}(A, M)$ be the set of all quadratic forms $A \rightarrow M$; this is an abelian group by $\left(\alpha_{e}, \alpha_{e e}\right)+\left(\beta_{e}, \beta_{e e}\right)=\left(\alpha_{e}+\beta_{e}, \alpha_{e e}+\beta_{e e}\right)$. Hence we obtain the quadratic

\section{Hom-functor}

$$
\operatorname{Hom}_{\mathbb{Z}}: \mathbf{M}(\mathbb{Z})^{o p} \times \mathbf{Q M}(\mathbb{Z}) \rightarrow \mathbf{A b}
$$

which generalizes the classical Hom-functor of abelian groups. On the other hand we have the quadratic tensor product

$$
\otimes_{\mathbb{Z}}: \mathbf{M}(\mathbb{Z}) \times \mathbf{Q M}(\mathbb{Z}) \rightarrow \mathbf{A b}
$$

which generalizes the classical tensor product of abelian groups. Here $A \otimes_{\mathbb{Z}} M$ is the abelian group generated by the symbols $a \otimes m,[a, b] \otimes n$ with $a, b \in A, m \in M_{e}$, $n \in M_{e e}$. The relations are

$$
\left\{\begin{array}{l}
(a+b) \otimes m=a \otimes m+b \otimes m+[a, b] \otimes H(m), \\
{[a, a] \otimes n=a \otimes P(n),}
\end{array}\right.
$$

where $a \otimes m$ is linear in $m$ and $[a, b] \otimes n$ is linear in each variable $a, b$ and $n$. We point out that $A \otimes_{\mathbb{Z}} M$ and $\operatorname{Hom}_{\mathbb{Z}}(A, M)$ are additive in $M$.

(8.3) Lemma. Let $A$ be a finitely generated free abelian group. Then one has the isomorphism

$$
\chi:{ }^{\#} A \otimes_{\mathbb{Z}} M \cong \operatorname{Hom}_{\mathbb{Z}}(A, M)
$$

which is natural in $A$. Here we set ${ }^{\#} A=\operatorname{Hom}(A, \mathbb{Z})$.

Proof. For $a, b \in \operatorname{Hom}_{\mathbb{Z}}(A, \mathbb{Z})$ let $\chi(a \otimes m)=\alpha=\left(\alpha_{e}, \alpha_{e e}\right)$ be given as follows: $(x, y \in A), \alpha_{e}(x)=a(x) m+(a(x)(a(x)-1) / 2) P H(m)$, and $\alpha_{e e}(x, y)=$ $a(x) a(y) H(m)$. Moreover $\chi([a, b] \otimes n)=\beta=\left(\beta_{e}, \beta_{e e}\right)$ is defined by $\beta_{e}(x)=$ $a(x) b(x) P(n)$, and $\beta_{e e}(x, y)=a(x) b(y) n+a(y) b(x) T n$.

Homotopy groups of spheres yield for $m<3 n-2$ the quadratic $\mathbb{Z}$-modules

$$
\Pi_{m}\left\{S^{n}\right\}=\left(\Pi_{m}\left(S^{n}\right) \stackrel{H}{\rightarrow} \Pi_{m}\left(S^{2 n-1}\right) \stackrel{P}{\rightarrow} \Pi_{m}\left(S^{n}\right)\right)
$$

where $H=\gamma_{2}$ is the Hopf invariant and where $P$ is induced by the Whitehead product square $\left[\iota_{n}, \iota_{n}\right]$, that is $P(\alpha)=\left[\iota_{n}, \iota_{n}\right] \circ \alpha$. In $\Pi_{m}\left\{S^{n}\right\}$ we get the involution $T=H P-1=(-1)^{n}$.

(8.5) Lemma. Let $m<3 n-2$ and suppose $X$ has the homotopy type of a finite one point union of spheres $S^{n}, n \geq 2$. Then one has isomorphisms

$$
\Pi_{m}(X) \cong H_{n}(X) \otimes_{\mathbb{Z}} \Pi_{m}\left\{S^{n}\right\} \cong \operatorname{Hom}_{\mathbb{Z}}\left(H^{n}(X), \Pi_{m}\left\{S^{n}\right\}\right)
$$

which are natural in $X$.

Proof. Let $a, b \in \Pi_{n}(X) \cong H_{n}(X)$. Then the first isomorphism carries $a \otimes m$, resp. $[a, b] \otimes n$, to the compositions $a \circ m$, resp. $[a, b] \circ n$, where $[a, b]$ is the Whitehead product, $m \in \Pi_{m}\left(S^{n}\right), n \in \Pi_{m}\left(S^{2 n-1}\right)$. The second isomorphism is given by (8.3) since $H^{n}(X)={ }^{\#} H_{n}(X)$.

In addition homotopy groups of the special orthogonal groups yield the quadratic $\mathbb{Z}$-modules $(n \geq 2)$

$$
\Pi_{n-1}\left\{S O_{n}\right\}=\left(\Pi_{n-1} S O_{n} \rightarrow \mathbb{Z} \rightarrow \Pi_{n-1} S O_{n}\right)
$$

together with a map

$$
\mathcal{J}=\mathcal{J}_{n}: \Pi_{n-1}\left\{S O_{n}\right\} \rightarrow \Pi_{2 n-1}\left\{S^{n}\right\}
$$


in $\operatorname{QM}(\mathbb{Z})$. Here $\mathcal{J}_{e e}$ carries $1 \in \mathbb{Z}$ to $\iota_{2 n-1} \in \Pi_{2 n-1}\left(S^{2 n-1}\right) \cong \mathbb{Z}$ and $\mathcal{J}_{e}$ : $\Pi_{n-1} S O_{n} \rightarrow \Pi_{2 n-1} S^{n}$ is the $\mathcal{J}$-homomorphism so that $H$ in (8.6) coincides with $\mathcal{J}_{e e}^{-1} H \mathcal{J}_{e}$. Moreover $P$ in (8.6) carries $1 \in \mathbb{Z}$ to $\partial i_{n}$ where we use the boundary $\partial: \Pi_{n} S^{n} \rightarrow \Pi_{n-1}\left(S O_{n}\right)$ of the fibering $S^{n}=S O_{n+1} / S O_{n}$.

(8.8) Definition. Let $M$ be a quadratic $\mathbb{Z}$-module with $M_{e e} \cong \mathbb{Z}$ and let $A$ be a finitely generated free abelian group (i.e. a $\mathbb{Z}$-lattice). We call a quadratic form $\alpha$ : $A \rightarrow M$ unimodular if the product $\alpha_{e e}: A \times A \rightarrow M_{e e} \cong \mathbb{Z}$ yields an isomorphism $D_{\alpha}: A \cong{ }^{\#} A$ where $D_{\alpha}$ is defined by $D_{\alpha}(x)(y)=\left\langle D_{\alpha}(x), y\right\rangle=\alpha_{e e}(x, y)$ for $x, y \in A$.

(8.9) Remark. Let $A$ be a $\mathbb{Z}$-lattice. Then a pre- $n$-space in the sense of Wall [40] is the same as a quadratic form $\alpha: A \rightarrow \Pi_{n-1}\left\{S O_{n}\right\}$; moreover this is an $n$-space if $\alpha$ is unimodular. Wall shows that "almost closed differential $(n-1)$ connected $2 n$-manifolds $M$ " stand in 1-1 correspondence with " $n$-spaces $\alpha$ ", $n \geq 3$. The correspondence carries $M$ to $\alpha=\alpha_{M}$ where $\alpha_{e e}$ is the intersection form of $M$ and where the function $\alpha_{e}: H_{n} M \rightarrow \Pi_{n-1} S O_{n}$ assigns to $x \in \Pi_{n} M=H_{n} M$ the classifying map of the normal bundle of an embedded sphere representing $x$.

We now call two quadratic forms $\alpha: A \rightarrow M$ and $\beta: B \rightarrow M$ equivalent if there exists an isomorphism $y: A \cong B$ with $\alpha_{e e}(y \times y)= \pm \beta_{e e}$ and $\alpha_{e} y=\beta_{e}$.

(8.10) Lemma. Homotopy types of $(n-1)$-connected Poincaré complexes $M$ of dimension $2 n$ are in 1-1 correspondence with equivalence classes of unimodular quadratic forms $A \rightarrow \Pi_{2 n-1}\left\{S^{n}\right\}$ where $A$ is a $\mathbb{Z}$-lattice, $n \geq 2$.

We obtain the correspondence in (8.10) as follows. Let $f$ be the attaching map of $M=\dot{M} \cup_{f} e^{2 n}$ and let $A=H_{n} M=H_{n} \dot{M}$. Then we have by (8.5) the natural isomorphism

$$
\Pi_{2 n-1}(\dot{M}) \cong A \otimes_{\mathbb{Z}} \Pi_{2 n-1}\left\{S^{n}\right\} \cong \operatorname{Hom}\left({ }^{\#} A, \Pi_{2 n-1}\left\{S^{n}\right\}\right)
$$

which carries $f$ to the quadratic form $\beta_{M}: B \rightarrow \Pi_{2 n-1}\left\{S^{n}\right\}$ with $B={ }^{\#} A=$ $H^{n}(\dot{M})$. We call $\beta_{M}$ the extended cup product of $M$. One can check that $\beta_{M}=\left(\beta_{e}, \beta_{e e}\right)$ coincides with the invariant considered by Kervaire-Milnor in [28]. In fact $\beta_{e e}$ is the cup product pairing $H^{n} M \times H^{n} M \rightarrow H^{2 n}(M)=\mathbb{Z}$ where $H^{n} M=$ $B$, that is, $\beta_{e e}(x, y)=\langle x \cup y,[M]\rangle$, and $\beta_{e}$ is the cohomology operation considered by Kervaire-Milnor, see 8.2 [28]. Wall in Lemma 8 [40] shows that for a closed differential $(n-1)$-connected $2 n$-manifold $M$ we have

$$
\mathcal{J}_{*} \alpha_{M}=D_{M}^{*} \beta_{M}=\delta_{M}
$$

where $\mathcal{J}$ is the map in (8.7) and where $D_{M}: H_{n} M \rightarrow H^{n} M$ is the inverse of the Poincaré duality isomorphism $\cap[M]: H^{n}(M) \cong H_{n} M$. Here $\cap[M]$ can be identified with ${ }^{\#} D_{\beta}=\operatorname{Hom}\left(D_{\beta}, \mathbb{Z}\right)$ : ${ }^{\#} A \rightarrow A$ where $D_{\beta}$ is given via $\beta_{e e}$ as in (8.8); this is readily checked by the formula $\langle x \cup y,[M]\rangle=\langle x, y \cap[M]\rangle$. Now the correspondence in (8.10) carries $M$ to the quadratic form

$$
\delta_{M}=D_{M}^{*} \beta_{M}=\left({ }^{\#} D_{\beta}^{-1}\right)^{*} \beta_{M}: A \rightarrow \Pi_{2 n-1}\left\{S^{n}\right\}
$$

which we call the extended intersection form of $M$; in fact, $\delta_{e e}$ in $\delta_{M}=\left(\delta_{e}, \delta_{e e}\right)$ is the classical intersection form. 
Proof of (8.10). Let $u: M \rightarrow N$ be a homotopy equivalence with $u_{*}[M]=\varepsilon[N]$, $\varepsilon \in\{+1,-1\}$. Then we get the restriction $v: \dot{M} \rightarrow \dot{N}$ of $u$ with $v_{*} f=\varepsilon f^{\prime}$ where $f$ and $f^{\prime}$ are the attaching maps. Hence by (1) above we get for $x=H_{n}(v)$ the equation $\left({ }^{\#} x\right)^{*} \beta=\varepsilon \beta^{\prime}$ with $\beta=\beta_{M}$ and $\beta^{\prime}=\beta_{N}$. In particular $\beta_{e e}\left({ }^{\#} x \times{ }^{\#} x\right)=$ $\varepsilon \beta_{e e}^{\prime}$ and hence $D^{\prime}=\varepsilon x D^{\#} x$ with $D^{\prime}=D_{\beta^{\prime}}$ and $D=D_{\beta}$. Therefore we get for $\delta=\delta_{M}, \delta^{\prime}=\delta_{N}$ via (3) the equations $\left(y=x^{-1}\right)$

$$
\begin{aligned}
\delta_{e} & =\beta_{e}^{\#} D^{-1}=\beta_{e}^{\#}\left(\varepsilon y D^{\prime \#} y\right)^{-1} \\
& =\left(\varepsilon \beta_{e}^{\prime \#} y\right)^{\#}\left(\varepsilon y D^{\prime \#} y\right)^{-1} \\
& \left.=\beta_{e}^{\prime}{ }^{\#} D^{\prime}\right)^{-1} y^{-1}=\delta_{e}^{\prime} y^{-1} .
\end{aligned}
$$

On the other hand one gets in a similar way $\delta_{e e}=\varepsilon \delta_{e e}^{\prime}\left(y^{-1} \times y^{-1}\right)$. Hence $\delta_{e}=\delta_{e}^{\prime} x$ and $\delta_{e e}=\varepsilon \delta_{e e}^{\prime}(x \times x)$ so that $x$ is an equivalence.

The $\mathcal{J}$-homomorphism (8.12) induces the homomorphism

$$
\mathcal{J}_{*}: \operatorname{Hom}_{\mathbb{Z}}\left(H_{n}, \Pi_{n-1}\left\{S O_{n}\right\}\right) \rightarrow \operatorname{Hom}_{\mathbb{Z}}\left(H_{n}, \Pi_{2 n-1}\left\{S^{n}\right\}\right)
$$

which clearly is injective if $\mathcal{J}_{e}$ is injective. Hence $\alpha_{M}$ in (8.10) (2) is determined by $\delta_{M}$ in case $\mathcal{J}_{e}$ is injective; this is actually the case for $n=$ odd or $n \equiv 6$ (8), see (8.21) below. Therefore we get:

(8.12) Remark. Let $n$ be odd or $n \equiv 6$ (8), $n \geq 3$, and let $M_{1}, M_{2}$ be differential $(n-1)$-connected $2 n$-manifolds of the same homotopy type. Then for some manifold $T$ homeomorphic to $S^{2 n}, M_{1}$ is diffeomorphic to $M_{2} \# T$. If $n=3,6, M_{1}$ is diffeomorphic to $M_{2}$. This corresponds to Theorem 5 of Wall [40], where Wall, however, does not treat the case $n \equiv 1$ (8). In this case $\mathcal{J}_{e}$ is still injective as follows from Theorem 1.1 of Adams [1].

Next we determine the group of homotopy equivalences of an $(n-1)$-connected Poincaré complex $M$ of dimension $2 n$ in terms of the classifying invariant $\delta_{M}$ above. For this we need the group of automorphisms, Aut $(\delta)$, of a quadratic form $\delta=\left(\delta_{e}, \delta_{e e}\right): A \rightarrow N$. This is the subgroup

$$
\operatorname{Aut}(\delta) \subset \operatorname{Aut}(A)
$$

consisting of all automorphisms $x: A \cong A$ that satisfy $\delta_{e} x=\delta_{e}$ and $\delta_{e e}(x \times x)=\varepsilon \delta_{e e}$ with $\varepsilon \in\{+1,-1\}$. We set $\varepsilon=\operatorname{deg}(x)$. If $N$ is an abelian group we have $\delta_{e e}=0$ so that the second equation is redundant. On the other hand if $H: N_{e} \rightarrow N_{e e}$ is injective we see by the second equation in $(8.2)(9)$ that the first equation is redundant; that is, in this case $\operatorname{Aut}(\delta)=\left\{x ; \delta_{e e}(x \times x)= \pm \delta_{e e}\right\}$.

(8.14) Theorem. Let $M$ be an $(n-1)$-connected Poincaré complex of dimension $2 n$ and let $M$ be $\Sigma$-reducible; that is $\Sigma \delta_{e}=0$ where $\delta_{e}: H_{n}(M) \rightarrow \Pi_{2 n-1} S^{n}$ is given by the extended intersection form $\delta_{M}=\left(\delta_{e}, \delta_{e e}\right)$ of $M$. Then the fundamental extension (1.3) has the form

$$
H_{n}(M) \otimes T_{n} \longmapsto \mathcal{E}(M) \stackrel{H_{n}}{\rightarrow} \operatorname{Aut}\left(\delta_{M}\right) .
$$

Here $T_{n}=$ Tor $\Pi_{2 n+1}\left(S^{n+1}\right)$ is the group in (6.2) and $H_{n}(M) \otimes T_{n}$ is an $\operatorname{Aut}\left(\delta_{M}\right)$ module by $x \cdot(a \otimes t)=\operatorname{deg}(x) \cdot x(a) \otimes t$ for $x \in \operatorname{Aut}\left(\delta_{M}\right), a \in H_{n}(M)$ and $t \in T_{n}$. The homomorphism $H_{n}$ carries an element in $\mathcal{E}(M)$ to the induced homomorphism in homology. The fundamental extension for $\mathcal{E}(M)$ above is split if in addition $n$ is odd or $n \in\{2,6\}$. 
Proof. The suspension $\Sigma$ induces a map $(m \leq 3 n-2, n \geq 2)$

$$
\Sigma=(\Sigma, 0): \Pi_{m}\left\{S^{n}\right\} \rightarrow \Pi_{m+1}\left\{S^{n+1}\right\}
$$

in $\mathrm{QM}(\mathbb{Z})$ where $\Sigma_{e}=\Sigma$ is the ordinary suspension and where $\Sigma_{e e}=0$ is trivial, compare (8.4). Now $\Sigma$ in (1) induces via (8.5) the suspension homomorphism on $\Pi_{m}(X)$ which is the composition

$$
\Pi_{m}(X) \cong H_{n}(X) \otimes_{\mathbb{Z}} \Pi_{m}\left\{S^{n}\right\} \stackrel{\sigma \otimes \Sigma}{\rightarrow} H_{n+1}(\Sigma X) \otimes_{\mathbb{Z}} \Pi_{m+1}\left\{S^{n+1}\right\} \cong \Pi_{m+1}(\Sigma X) .
$$

Here $\sigma$ is the homology suspension $\sigma: H_{n}(X) \cong H_{n+1}(\Sigma X)$. Therefore we see by (8.5) that $M$ is $\Sigma$-reducible if and only if $\Sigma \delta_{e}=0$. As in the proof of (8.10) we get

$$
\operatorname{Aut}\left(\delta_{M}\right) \cong \mathcal{E}(\stackrel{\bullet}{M}, \pm f)
$$

Here we use the naturality of the isomorphisms (8.5) and (8.10) (1). By (3) and Theorem (3.5) we then obtain the fundamental extension in (8.14). We now deduce the splitting in (8.14) from Theorem (5.2). For this we consider the inclusion (5.1) which via (8.5) corresponds to the inclusion $\left(A=H_{n} \dot{M}\right)$

$$
A \otimes_{\mathbb{Z}} \Sigma \Pi_{2 n}\left\{S^{n}\right\} \stackrel{1 \otimes i}{\rightarrow} A \otimes_{\mathbb{Z}} \Pi_{2 n+1}\left\{S^{n+1}\right\}
$$

where $i$ is the inclusion of the image of $\Sigma$ in (1). In case there is a retraction $r$ in $\operatorname{QM}(\mathbb{Z})$, for which

$$
\Sigma \Pi_{2 n}\left\{S^{n}\right\} \stackrel{i}{\rightarrow} \Pi_{2 n+1}\left\{S^{n+1}\right\} \stackrel{r}{\rightarrow} \Sigma \Pi_{2 n}\left\{S^{n}\right\}
$$

is the identity, we see that (4) admits the retraction $1 \otimes r$ which is natural in A. Hence in this case the fundamental extension in (8.14) is split by (5.2). The retraction $r$ in (5) exists if and only if there is a retraction $r_{e}$ of the inclusion $\Sigma \Pi_{2 n}\left(S^{n}\right) \subset \Pi_{2 n+1} S^{n+1}$ for which $r_{e}\left[\iota_{n+1}, \iota_{n+1}\right]=0$. This is the case if and only if $n$ is odd or $n \in\{2,6\}$, see [39].

The construction of the retraction $1 \otimes r$ of (4) in the proof above illustrates indeed the usefulness of the quadratic tensor product in (8.2). Our approach avoids the choice of a basis in $H_{n} M$; such a choice would imply a mess of equations as one can find them in this context often in the literature. Also the following considerations show the clear advantages of our approach. We describe in more detail the properties of the quadratic $\mathbb{Z}$-modules $\Pi_{2 n-1}\left\{S^{n}\right\}$ and $\Pi_{n-1}\left\{S O_{n}\right\}$. For 
this we introduce the following list of indecomposable quadratic $\mathbb{Z}$-modules.

$$
\begin{aligned}
& \mathbb{Z}^{\otimes}=(\mathbb{Z} \stackrel{(1,1)}{\rightarrow} \mathbb{Z} \oplus \mathbb{Z} \stackrel{(1,1)}{\rightarrow} \mathbb{Z}), \\
& \mathbb{Z}^{S}=(\mathbb{Z} \stackrel{2}{\rightarrow} \mathbb{Z} \stackrel{1}{\rightarrow} \mathbb{Z}), \\
& \mathbb{Z}^{\Lambda}=(0 \rightarrow \mathbb{Z} \rightarrow 0), \\
& \mathbb{Z}_{r}^{\Lambda}=\left(\mathbb{Z} / 2^{r} \stackrel{0}{\rightarrow} \mathbb{Z}^{2^{r-1}} \rightarrow \mathbb{Z} / 2^{r}\right), \\
& \mathbb{Z}^{\Gamma}=(\mathbb{Z} \stackrel{1}{\rightarrow} \mathbb{Z} \stackrel{2}{\rightarrow} \mathbb{Z})
\end{aligned}
$$

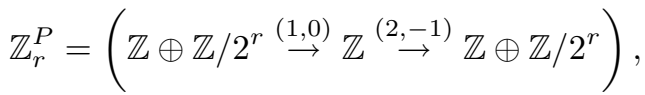

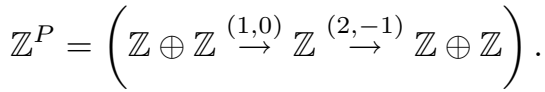

We have obvious quotient maps $\mathbb{Z}^{P} \rightarrow \mathbb{Z}_{r}^{P} \rightarrow \mathbb{Z}^{\Gamma}$ and $\mathbb{Z}_{r}^{\Lambda} \rightarrow \mathbb{Z}^{\Lambda}$ in $\mathbf{Q M}(\mathbb{Z})$. Some of the quadratic $\mathbb{Z}$-modules in (8.15) correspond to well-known quadratic functors $\mathbf{A b} \rightarrow \mathbf{A b}$ since there are natural isomorphisms $(A \in \mathbf{A b})$

$$
\begin{aligned}
\otimes^{2}(A) & =A \otimes_{\mathbb{Z}} \mathbb{Z}^{\otimes}=A \otimes A, \\
S^{2}(A) & =A \otimes_{\mathbb{Z}} \mathbb{Z}^{S}=A \otimes A /\{a \otimes b-b \otimes a\}, \\
\Lambda^{2}(A) & =A \otimes_{\mathbb{Z}} \mathbb{Z}^{\Lambda}=A \otimes A /\{a \otimes a\}, \\
\hat{\otimes}^{2}(A) & =A \otimes_{\mathbb{Z}} \mathbb{Z}_{1}^{\Lambda}=A \otimes A /\{a \otimes b+b \otimes a\}, \\
\Gamma(A) & =A \otimes_{\mathbb{Z}} \mathbb{Z}^{\Gamma}, \\
P^{2}(A) & =A \otimes_{\mathbb{Z}} \mathbb{Z}^{P}=\Delta(A) / \Delta(A)^{3} .
\end{aligned}
$$

Here $S^{2}(A)$ and $\Lambda^{2}(A)$ are the symmetric resp. exterior square. Moreover $\Gamma$ is the quadratic functor of J. H. C. Whitehead [41] which is part of the free commutative ring with divided powers $[20, \S 13]$. Moreover $P^{2}(A)$ is the polynomial construction given by the augmentation ideal $\Delta(A)$ and its third power $\Delta(A)^{3}$ in the groupring $\mathbb{Z}[A]$ of the group $A$. The sum $\mathbb{Z}^{\otimes} \oplus \mathbb{Z}^{P}$ is actually the universal quadratic $\mathbb{Z}$-module, see [13].

(8.17) Lemma. There are indecomposable quadratic $\mathbb{Z}$-modules $S_{n}^{Q}, O_{n}^{Q}$ as in (8.15) and abelian groups $S_{n}^{L}, O_{n}^{L}$ such that for $n \geq 2$ one has isomorphisms in $\mathrm{QM}(\mathbb{Z})$

$$
\Pi_{2 n-1}\left\{S^{n}\right\}=S_{n}^{Q} \oplus S_{n}^{L}, \quad \Pi_{n-1}\left\{S O_{n}\right\}=O_{n}^{Q} \oplus O_{n}^{L} .
$$

More precisely we obtain the following lists in which we describe $S_{n}^{Q}, O_{n}^{Q}, S_{n}^{L}$, $O_{n}^{L}$ respectively. We write "g.d.s" for "generates a direct summand" and $\Pi_{n-1}^{S}$ denotes the stable $(n-1)$-stem. Using results of Toda in [39] and Kervaire [29] (see 
Wall [40, p. 171]) we get:

$$
\Pi_{2 n-1}\left\{S^{n}\right\}= \begin{cases}\mathbb{Z}^{\Gamma}, & n=2, \\ \mathbb{Z}_{2}^{P} \oplus \mathbb{Z} / 3, & n=4, \\ \mathbb{Z}_{3}^{P} \oplus \mathbb{Z} / 15, & n=8, \\ \mathbb{Z}^{\Lambda} \oplus \mathbb{Z} / 2, & n=3,7, \\ \mathbb{Z}^{S} \oplus \Pi_{n-1}^{S}, & n \text { even, } n \notin\{2,4,8\}, \\ \mathbb{Z}_{1}^{\Lambda} \oplus \Pi_{n-1}^{S}, & n \text { odd, } n \notin\{3,7\},\left[\iota_{n}, \iota_{n}\right] \text { g.d.s, } \\ \mathbb{Z}_{r}^{\Lambda} \oplus \Pi_{n-1}^{S} /\left\{\sum \alpha_{n}\right\}, & n \text { odd, } n \notin\{3,7\}, \\ & {\left[\iota_{n}, \iota_{n}\right]=2^{r-1} \alpha_{n}, \alpha_{n} \text { g.d.s. }}\end{cases}
$$

The divisibilities of the Whitehead square in $(K)$ is relevant with respect to the Arf invariant problem, see [31].

$$
\Pi_{n-1}\left\{S O_{n}\right\}= \begin{cases}\mathbb{Z}^{\Gamma}, & n=2, \\ \mathbb{Z}^{P}, & n=4,8, \\ \mathbb{Z}^{\Lambda} & n=3,7, \\ \mathbb{Z}^{S} \oplus \Pi_{n-1} S O, & n \text { even, } n \notin\{2,4,8\}, \\ \mathbb{Z}_{1}^{\Lambda} \oplus \Pi_{n-1} S O, & n \text { odd, } n \notin\{3,7\} .\end{cases}
$$

For $n=4$ see Lemma 20.10 in [32]. We recall that by results of Bott [16] we have for $n \geq 2$ :

$$
\Pi_{n-1} S O= \begin{cases}\mathbb{Z} / 2, & n \equiv 1,2(8), \\ 0, & n \equiv 3,5,6,7(8), \\ \mathbb{Z}, & n \equiv 0(4) .\end{cases}
$$

There is a canonical inclusion $i_{n}^{Q}: O_{n}^{Q} \subset S_{n}^{Q}$ which is the identity except for the case $(K)$ in (8.18). Moreover we have the homomorphism $i_{n}^{L}: O_{n}^{L} \rightarrow S_{n}^{L}$ which is induced by the stable $\mathcal{J}$-homomorphism $\Pi_{n-1} S O \rightarrow \Pi_{n-1}^{S}$. Now the map $\mathcal{J}_{n}$ between quadratic $\mathbb{Z}$-modules in (8.7) is given by $(n \notin\{2,4,8\})$

$$
\mathcal{J}_{n}: \Pi_{n-1}\{S O\}=O_{n}^{Q} \oplus O_{n}^{L} \stackrel{i_{n}^{Q} \oplus i_{n}^{L}}{\rightarrow} S_{n}^{Q} \oplus S_{n}^{L}=\Pi_{2 n-1}\left\{S^{n}\right\}
$$

For $n \in\{2,4,8\}$ the map

$$
\mathcal{J}_{n}: \Pi_{n-1}\{S O\}=O_{n}^{Q} \rightarrow S_{n}^{Q} \oplus S_{n}^{L}=\Pi_{2 n-1}\left\{S^{n}\right\}
$$

is the canonical surjection. Here $O_{n}^{Q} \rightarrow S_{n}^{Q}$ is the identity for $n=2$ and the quotient map for $n \in\{4,8\}$; the second coordinate $O_{n}^{Q} \rightarrow S_{n}^{L}$ is given by $(1,2): \mathbb{Z} \oplus \mathbb{Z} \rightarrow S_{n}^{L}$ for $n \in\{4,8\}$.

(8.22) Lemma. Each $\Sigma$-reducible $(n-1)$-connected Poincaré complex of dimension $2 n$ has the homotopy type of a closed manifold.

Proof. We obtain the suspension

$$
\Sigma: \Pi_{2 n-1}\left\{S^{n}\right\}=S_{n}^{Q} \oplus S_{n}^{L} \rightarrow \Pi_{n-1}^{S}
$$

by dividing out the image of $P$ in $S_{n}^{Q}$, see (8.14)(1). This shows by inspection that $\operatorname{Ker}(\Sigma) \subset$ image $\mathcal{J}_{n}$. Hence the lemma is a consequence of $(8.10)(2)$. 
On the other hand we have $\Sigma \mathcal{J}_{n}=0$ if $\Pi_{n-1} S O=0$. Therefore $\operatorname{Ker}(\Sigma)=$ image $\mathcal{J}_{n}$ in this case and we get by (8.20) the proposition in (3.3).

Since the quadratic Hom functor is additive in the second variable we see that the extended intersection form $\delta_{M}$ and the Wall invariant $\alpha_{M}$ in (8.10) yield by (8.17) quadratic forms

$$
\left\{\begin{array}{l}
\delta_{M}^{Q}: H_{n} M \rightarrow S_{n}^{Q}, \text { resp. } \\
\alpha_{M}^{Q}: H_{n} M \rightarrow O_{n}^{Q}
\end{array}\right.
$$

which we call the quadratic part and homomorphisms between abelian groups

$$
\left\{\begin{array}{l}
\delta_{M}^{L}: H_{n} M \rightarrow S_{n}^{L}, \text { resp. } \\
\alpha_{M}^{L}: H_{n} M \rightarrow O_{n}^{L}
\end{array}\right.
$$

which we call the linear part. The automorphism group (8.13) then satisfies

(8.24) Proposition. Let $M$ be an $(n-1)$-connected Poincaré complex of dimension $2 n$ with extended intersection form $\delta_{M}$ and attaching map $f$. Then we have isomorphisms of groups

$$
\mathcal{E}(\dot{M}, \pm f)=\operatorname{Aut}\left(\delta_{M}\right)=\operatorname{Aut}\left(\delta_{M}^{Q}\right) \cap \operatorname{Aut}\left(\delta_{M}^{L}\right) .
$$

Clearly we assume $H_{n} M \neq 0$. The proposition follows by similar arguments as in the proof of (8.10). Kahn [26], [27] incorrectly describes the quadratic part, resp. linar part, of $\delta_{M}=\mathcal{J} \alpha_{M}$ so that therefore his definition of $\operatorname{Aut}(\mu, c)$ in his main result is also incorrect; the correct definition would be (8.24).

For the computation of $\mathcal{E}(M \mid \dot{M})$ we need the following definitions and facts.

(8.25) Definition. Let $A$ be an abelian group and let $M$ be a quadratic $\mathbb{Z}$-module. Then one has the natural homomorphism

$$
A \otimes_{\mathbb{Z}} M \stackrel{H}{\rightarrow} A \otimes A \otimes M_{e e} \stackrel{P}{\rightarrow} A \otimes_{\mathbb{Z}} M
$$

with

$$
\begin{aligned}
H(a \otimes m) & =a \otimes a \otimes H(m), \\
H([a, b] \otimes n) & =a \otimes b \otimes n+b \otimes a \otimes T(n), \\
P(a \otimes b \otimes n) & =[a, b] \otimes n,
\end{aligned}
$$

where $T=H P-1$ is the involution.

Let $\Sigma X$ be a one point union of spheres $S^{n}$ such that $H_{n} \Sigma X=A$. Then we have for $n \leq 3 n-2$ the commutative diagram

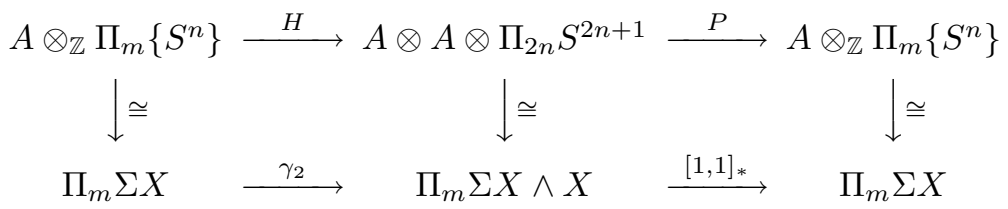

where we use the isomorphism (8.5). The diagram shows that $H$ in (8.25) corresponds to the James-Hopf invariant $\gamma_{2}$ and that $P$ corresponds to the map induced by the Whitehead product square $[1,1]$, see $\S 2$. The Hopf map $\eta_{m} \in \Pi_{m+1} S^{m}=$ $\mathbb{Z} / 2, m \geq 3$, is a generator which induces the following maps between quadratic $\mathbb{Z}$-modules, where $\Sigma \Pi_{2 n-1}\left(S^{n}\right)$ is a subgroup of $\Pi_{2 n} S^{n+1}$.

$$
\Pi_{2 n-1}\left\{S^{n}\right\} \stackrel{\eta_{2 n-1}^{*}}{\longrightarrow} \Pi_{2 n}\left\{S^{n}\right\} \stackrel{\left(\eta_{n}\right)_{*}}{\leftarrow} \mathbb{Z} / 2 \otimes \Sigma \Pi_{2 n-1}\left(S^{n}\right) .
$$


We use these maps in $\mathbf{Q M}(\mathbb{Z})$ in the following result. As usual we also write $A=1_{A}$ for the identity of $A$.

(8.28) Theorem. Let $n \geq 3$ and let $M$ be an $(n-1)$-connected Poincaré complex of dimension $2 n$ with $A=H_{n} M$ and attaching map $f_{M} \in A \otimes_{\mathbb{Z}} \Pi_{2 n-1}\left\{S^{n}\right\} \cong$ $\Pi_{2 n-1}(\dot{M})$. Then the module $\mathcal{E}(M \mid \dot{M})$ is algebraically given by the isomorphism

$$
\mathcal{E}(M \mid \dot{M}) \cong A \otimes \Pi_{2 n}\left\{S^{n}\right\} / \mathcal{J}
$$

where $\mathcal{J}$ is the following subgroup of $A \otimes_{\mathbb{Z}} \Pi_{2 n}\left\{S^{n}\right\} \cong \Pi_{2 n}(\dot{M})$. The subgroup $\mathcal{J}$ is generated by the element $\left(A \otimes \eta_{2 n-1}^{*}\right)\left(f_{M}\right)$ and by all elements

$$
P(A \otimes \xi) H\left(f_{M}\right)+\left(A \otimes \eta_{n_{*}}\right)(\xi \otimes \Sigma)\left(f_{M}\right)
$$

with $\xi \in \operatorname{Hom}(A, A \otimes \mathbb{Z} / 2)$. Here we use the homomorphisms

$$
\begin{gathered}
A \otimes_{\mathbb{Z}} \Pi_{2 n-1}\left\{S^{n}\right\} \stackrel{H}{\rightarrow} A \otimes A \stackrel{A \otimes \xi}{\rightarrow} A \otimes A \otimes \mathbb{Z} / 2 \stackrel{P}{\rightarrow} A \otimes_{\mathbb{Z}} \Pi_{2 n}\left\{S^{n}\right\}, \\
A \otimes_{\mathbb{Z}} \Pi_{2 n-1}\left\{S^{n}\right\} \stackrel{\xi \otimes \Sigma}{\rightarrow} A \otimes \mathbb{Z} / 2 \otimes \Sigma \Pi_{2 n-1} S^{n} \stackrel{A \otimes \eta_{n_{*}}}{\rightarrow} A \otimes_{\mathbb{Z}} \Pi_{2 n}\left\{S^{n}\right\} .
\end{gathered}
$$

A description of $\mathcal{E}(M \mid \dot{M})$ in terms of quadratic Hom-functors is also possible by use of the isomorphism in (8.5).

Proof of (8.28). We use the description of $\mathcal{J}$ in (2.2) where

$$
\xi \in[\Sigma \dot{M}, \dot{M}]=\operatorname{Hom}\left(A, A \otimes \Pi_{n+1} S^{n}\right) .
$$

Here $\eta_{n} \in \Pi_{n+1} S^{n}=\mathbb{Z} / 2$ is the generator. We have the formula

$$
\begin{aligned}
\nabla(1, f)(\xi) & =\xi \circ(\Sigma f)+[\xi, 1] \Sigma \gamma_{2} f \\
& =\xi \circ(\Sigma f)+[1,1]_{*}(A \wedge \xi) \Sigma \gamma_{2} f
\end{aligned}
$$

Now using (8.26) we obtain the description of $\mathcal{J}$ in (8.28).

For various $n$ the group $\mathcal{E}(M \mid \dot{M})$ in (8.28) can be computed in a better way. We do this in the next section for $n \in\{2,4,8\}$.

\section{MANifolds CONNECTED With Hopf Maps}

We consider $(n-1)$-connected Poincaré complexes of dimension $2 n$ for $n \in$ $\{2,4,8\}$. These are the cases for which the Hopf invariant problem is relevant. Moreover each such Poincaré complex has the homotopy type of a topological manifold. As a first example we consider the projective planes. Let $\mathbb{R} P_{2}, \mathbb{C} P_{2}, \mathbb{H} P_{2}$ be the real, resp. complex, resp. quaternionic projective plane and let $\mathcal{C} a$ be the Cayley plane. Recall that $\mathcal{E}(M)$ in Top/ $\simeq$ is defined with respect to maps which need not be base point preserving; let $\mathcal{E}(M)^{*}$ be the corresponding group for basepoint preserving maps, i.e. in Top* $/ \simeq$.

$$
\begin{aligned}
\mathcal{E}\left(\mathbb{R} P_{2}\right) & =0, \quad \mathcal{E}\left(\mathbb{R} P_{2}\right)^{*}=\mathbb{Z} / 2, \\
\mathcal{E}\left(\mathbb{C} P_{2}\right) & =\mathbb{Z} / 2, \\
\mathcal{E}\left(\mathbb{H} P_{2}\right) & =\mathbb{Z} / 2, \\
\mathcal{E}(\mathcal{C} a) & =\mathbb{Z} / 2 \oplus \mathbb{Z} / 2 .
\end{aligned}
$$

For $\mathbb{R} P_{2}$ see [9, IIIB.18]. Moreover $\mathcal{E}\left(\mathbb{C} P_{2}\right)$ is a special case of (9.3) below since for $M=\mathbb{C} P_{2}$ we have $\mathcal{E}(M \mid \dot{M})=0$ and $\operatorname{Aut}\left(\delta_{M}\right)=\operatorname{Aut}(\mathbb{Z})=\mathbb{Z} / 2$. For the 
manifolds $M=\mathbb{H} P_{2}$ and $M=\mathcal{C} a$, however, the group Aut $\left(\delta_{M}\right)$ is trivial. In fact the attaching map of $M$ is the Hopf map $\nu_{4}$, resp. $\sigma_{8}$, which is the generator of $\mathbb{Z}$ in $\Pi_{2 n-1} S^{n}=\mathbb{Z} \oplus \mathbb{Z} / 2^{r} \oplus$ odd torsion, $n=2^{r}$. Hence the extended intersection form (the quadratic part) is given by $\delta_{M}^{Q}=\left(\delta_{e}, \delta_{e e}\right): \mathbb{Z} \rightarrow \mathbb{Z}_{r}^{P}$ with $\delta_{e}(1)=(1,0)$. Thus we get by $(8.2)(1)$

$$
\begin{aligned}
\delta_{e}(-1) & =-\delta_{e}(1)+P \alpha_{e e}(1,1) \\
& =-\delta_{e}(1)+P(1) \\
& =-(1,0)+(2,-1) \\
& =(1,-1) \in \mathbb{Z} \oplus \mathbb{Z} / 2^{r}
\end{aligned}
$$

so that $\delta_{e}(-1) \neq \delta_{e}(1)$. Therefore $-1 \notin \operatorname{Aut}\left(\delta_{M}\right)$. Now the computation of $\mathcal{E}(M)=$ $\mathcal{E}(M \mid \dot{M})$ is a consequence of (9.6) below. Compare also [43].

We need the definition of the characteristic element of a quadratic form, see for example p. 26 of [25].

(9.2) Definition. Let $A$ be a $\mathbb{Z}$-lattice and let $\beta: A \times A \rightarrow \mathbb{Z}$ be a symmetric bilinear map with odd determinant. Then there exists an element $\omega_{0} \in A$ with $\beta(x, x) \equiv \beta\left(x, \omega_{0}\right) \bmod 2$ for all $x \in A$. The element $\omega=\omega_{0} \otimes 1 \in A \otimes \mathbb{Z} / 2$ is well defined by $\beta$ and is called the characteristic element of $\beta$.

For example, let $\beta=\cup: H^{2} M \times H^{2} M \rightarrow \mathbb{Z}$ be the cup product pairing of a 1-connected 4-dimensional Poincaré complex; then the characteristic element of $\beta=\cup$ is the second Stiefel-Whitney class $\omega_{2} \in H^{2}(M, \mathbb{Z} / 2)=H^{2}(M) \otimes \mathbb{Z} / 2$ of $M$.

(9.3) Theorem. Let $M$ be a 1-connected 4-dimensional Poincaré complex. Then one has the split extension

$$
\operatorname{Ker} \omega_{2} \longmapsto \mathcal{E}(M) \rightarrow \operatorname{Aut}\left(\delta_{M}\right) .
$$

Here $\omega_{2}: H_{2}(M) \otimes \mathbb{Z} / 2 \rightarrow \mathbb{Z} / 2$ is given by the second Stiefel-Whitney class of $M$. Moreover Aut $\left(\delta_{M}\right)$ acts on Ker $\omega_{2}$ by $x \cdot(a \otimes 1)=x(a) \otimes 1$ for $x \in \operatorname{Aut}\left(\delta_{M}\right)$, $a \otimes 1 \in \operatorname{Ker} \omega_{2}$.

Proof. This result was recently proved by Cochran-Habegger in [18]. Originally $\mathcal{E}(M \mid \dot{M})$ was computed in [11]; clearly this group can be derived from Theorem (2.2) above. Quinn [34] obtained the formula $\mathcal{E}(M \mid \dot{M}) \cong \operatorname{Ker} \omega_{2}$, but his proof was not correct. Cochran-Habegger do this calculation again and also describe an intricate homotopy theoretic proof for the splitting. If $\omega_{2}=0$ we obtain a new and short proof for the splitting by (8.14) since then $M$ is $\Sigma$-reducible; in fact for $\omega_{2}=0$ the extension in (9.3) coincides with the one in (8.14). If $\omega_{2} \neq 0$ one can use results of Freedman to describe a splitting as follows:

Freedman [21] shows that there is a closed topological manifold $N$ which has the homotopy type of the Poincaré complex $M$. Let $\Pi_{0} \operatorname{Homeo}(N)$ be the group of isotopy classes of homeomorphisms of $N$. Then Quinn [34] proved that the natural homeomorphism

$$
H_{2}: \Pi_{0} \operatorname{Homeo}(N) \stackrel{\cong}{\rightrightarrows} \operatorname{Aut}\left(\delta_{N}\right)
$$

is an isomorphism; in fact, surjectivity follows from Freedman's Theorem (1.5) Addendum [21] and injectivity can also be proved along the lines of the proof of Kreck's result Theorem 1 in [30]. Using this isomorphism the canonical forgetful 
homomorphism $\Pi_{0} \operatorname{Homeo}(N) \rightarrow \mathcal{E}(N)$ yields the splitting for the extension in (9.3).

For a quadratic $\mathbb{Z}$-module $M$ and an abelian group $C$ let $M \otimes C$ be the quadratic $\mathbb{Z}$-module given by

$$
M \otimes C=\left(M_{e} \otimes C \stackrel{H \otimes 1}{\rightarrow} M_{e e} \otimes C \stackrel{P \otimes 1}{\rightarrow} M_{e} \otimes C\right) .
$$

Then the quadratic tensor product satisfies

$$
A \otimes_{\mathbb{Z}}(M \otimes C)=\left(A \otimes_{\mathbb{Z}} M\right) \otimes C .
$$

For example we have

$$
\mathbb{Z}^{P} \otimes \mathbb{Z} / 2=\mathbb{Z}_{r}^{P} \otimes \mathbb{Z} / 2=(\mathbb{Z} / 2 \oplus \mathbb{Z} / 2 \stackrel{(1,0)}{\rightarrow} \mathbb{Z} / 2 \stackrel{(0,1)}{\rightarrow} \mathbb{Z} / 2 \oplus \mathbb{Z} / 2)
$$

This quadratic $\mathbb{Z}$-module is part of the following commutative diagram in $\mathbf{Q M}(\mathbb{Z})$ with short exact rows and columns:

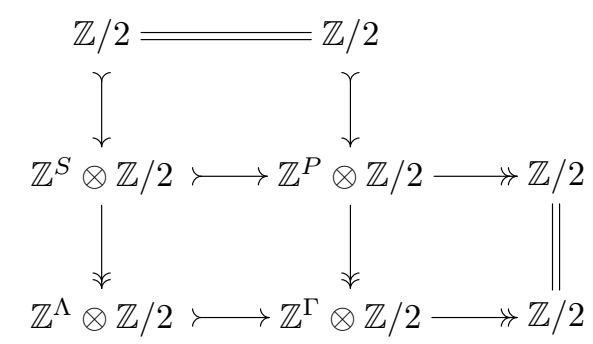

For a $\mathbb{Z}$-lattice $A$ the functor $A \otimes_{\mathbb{Z}^{-}}$, applied to this diagram, yields the following commutative diagram with exact rows and columns in $\mathbf{A b}$, see the notation in $(8.16)$.

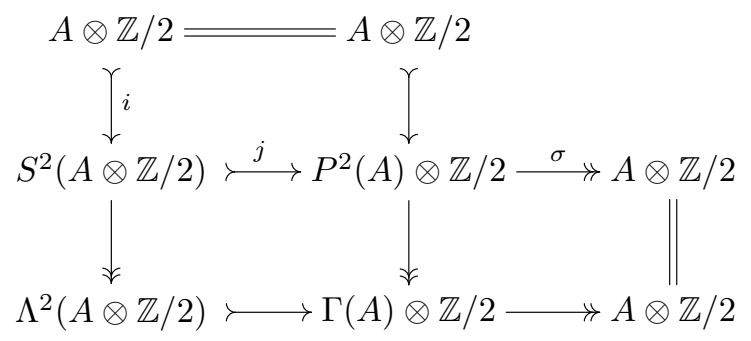

Here $i$ carries $y$ to $i(y)=y \cdot y=\{y \otimes y\}$ and $j$ corresponds to $P$ in (8.25), that is, $j(x \cdot y)=P(x \otimes y)$. For a homomorphism $\omega \in \operatorname{Hom}(A, \mathbb{Z} / 2)=\operatorname{Hom}(A \otimes \mathbb{Z} / 2, \mathbb{Z} / 2)$ we get the next push out diagram in $\mathbf{A b}$ with short exact rows; this diagram defines $E_{4}(\omega)$.

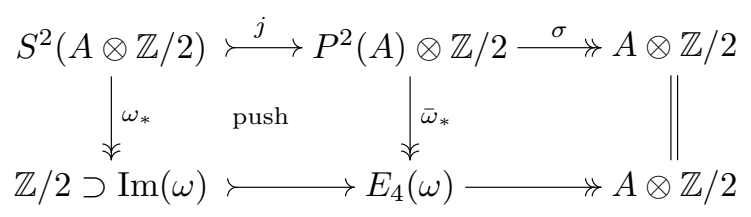


Here $\omega_{*}$ carries $\{x \otimes y\}$ to $\omega(x) \cdot \omega(y)$ for $x, y \in A$ where we use the ring structure of $\mathbb{Z} / 2=\mathbb{Z} / 2 \mathbb{Z}$. On the other hand we use $\omega$ also for the following push out diagram which defines $E_{8}(\omega)$ for $\omega \neq 0$.

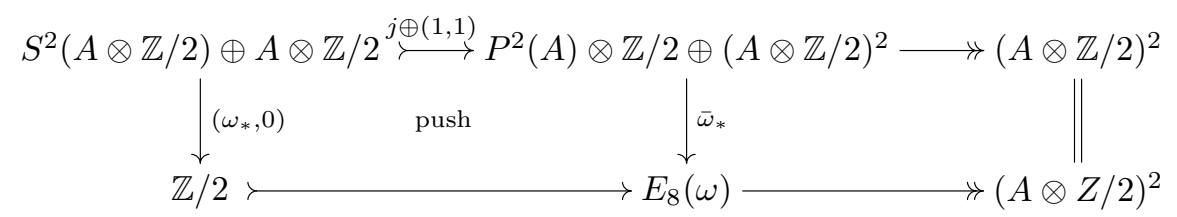

Here $\omega_{*}$ is defined as in $(3)$ and $(1,1)$ carries $x$ to $x \oplus x$. For $\omega=0$ we get the quotient map

$$
\bar{\omega}_{*}=\sigma \oplus 1 \oplus 1: P^{2}(A) \otimes \mathbb{Z} / 2 \oplus(A \otimes \mathbb{Z} / 2)^{2} \rightarrow E_{8}(0)=(A \otimes \mathbb{Z} / 2)^{3}
$$

where $\sigma$ is defined in (2). We point out that diagrams (3), (4) and (5) are in the obvious way diagrams of $\operatorname{Aut}(\omega)$-modules with $\operatorname{Aut}(\omega) \subset \operatorname{Aut}(A)$ defined in (8.13).

(9.5) Theorem. Let $n=4$ or $n=8$ and let $M$ be an $(n-1)$-connected Poincaré complex of dimension $2 n$ with extended intersection form $\delta_{M} \in \operatorname{Hom}_{\mathbb{Z}}\left(A, \Pi_{2 n-1}\left\{S^{n}\right\}\right)$ with $A=H_{n} M$. Then $\delta_{M}$ determines the element $\omega=\omega_{M} \in \operatorname{Hom}(A, \mathbb{Z} / 2)$ which is the characteristic class of the cup product pairing $\cup: H^{n} M \times H^{n} M \rightarrow \mathbb{Z}$. Moreover $\delta_{M}$ determines the element $\nu=\nu_{M} \in P^{2}(A) \otimes \mathbb{Z} / 2$ which is the reduction mod 2 of the attaching map of $M$. The map $\sigma$ in (9.4) (3) carries $\nu$ to the element $\sigma(\nu) \in A \otimes \mathbb{Z} / 2$ which is the Poincaré dual of $\omega$. With these data we have the fundamental extension

$$
E_{n}(\omega) /\left\{\bar{\omega}_{*}(\nu)\right\} \longmapsto \mathcal{E}(M) \rightarrow \operatorname{Aut}\left(\delta_{M}\right) .
$$

Here the group $E_{n}(\omega) /\left\{\bar{\omega}_{*}(\nu)\right\}$ is an $\operatorname{Aut}\left(\delta_{M}\right)$-module via the structure of $E_{n}(\omega)$ as an $\operatorname{Aut}(\omega)$-module; clearly $\operatorname{Aut}\left(\delta_{M}\right) \subset \operatorname{Aut}(\omega)$.

If $\omega \neq 0$ then also $\bar{\omega}_{*}(\nu) \neq 0$ since $\sigma(\nu) \neq 0$. This shows that one has isomorphisms of $\mathbb{Z} / 2$-vector spaces

$$
\mathcal{E}(M \mid \dot{M}) \cong \begin{cases}H_{n}(M) \otimes \mathbb{Z} / 2 & \text { if } n=4 \\ \left(H_{n}(M) \otimes \mathbb{Z} / 2\right)^{2} & \text { if } n=8, \omega \neq 0 \\ \left(H_{n}(M) \otimes \mathbb{Z} / 2\right)^{3} & \text { if } n=8, \omega=0\end{cases}
$$

This is also an isomorphism of $\operatorname{Aut}\left(\delta_{M}\right)$-modules in case $\omega=0$. We point out that the following three conditions are equivalent for $M$ in the theorem:

(i) the intersection form of $M$ is even,

(ii) the characteristic class of the intersection form vanishes, that is, $\omega=0$,

(iii) the reduction mod 2 of the suspended attaching map vanishes, that is, $\sigma(\nu)=$ 0.

Hence if $M$ is $\Sigma$-reducible then $\omega=0$ and $\bar{\omega}_{*}(\nu)=\sigma(\nu)=0$.

Proof of (9.5). Clearly $\delta_{M}$ determines the extended cup product and the attaching map $f_{M}$ by the isomorphisms in (8.10) (1) (2) (3). Recall that for $n \in\{4,8\}$ with $n=2^{r}$ we have

$$
\Pi_{2 n-1}\left\{S^{n}\right\}=\mathbb{Z}_{r}^{P} \oplus S_{n}^{L}
$$


where $S_{n}^{L}$ is odd torsion, see (8.18). Hence $f_{M}$ in (8.27) determines the quadratic part

$$
f_{M}^{Q} \in A \otimes \mathbb{Z}_{r}^{P}
$$

Here we have $A=H_{n} M$ and ${ }^{*} A=\operatorname{Hom}(A, \mathbb{Z})=H^{n}(M)$. By use of the following commutative diagram we see that $\sigma(\nu)$ is the Poincaré dual of $\omega$. Let $x \in \operatorname{Hom}(A, \mathbb{Z})$. Then we get by naturality the commutative diagram

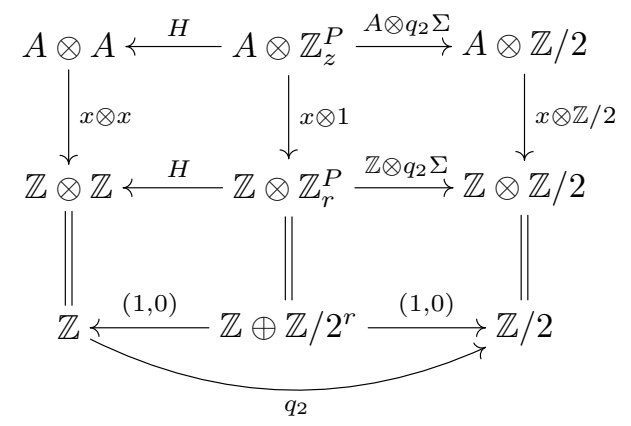

Here $q_{2} \Sigma$ is given by the composition

$$
q_{2} \Sigma=(1,0): \mathbb{Z} \oplus \mathbb{Z} / 2^{r} \stackrel{\Sigma}{\rightarrow} \mathbb{Z} / 2^{r+1} \stackrel{q_{2}}{\rightarrow} \mathbb{Z} / 2
$$

where $\Sigma=(1,2)$, since Ker $\Sigma=\operatorname{Im} P$. Now we know that the cup product $H^{n} M \times H^{n} M \rightarrow \mathbb{Z}$ satisfies the formula

$$
x \cup y=(x \otimes y) H\left(f_{M}^{Q}\right)
$$

where $f_{M}^{Q}$ is the quadratic part in (2). Hence by (3) and (1) we get

$$
\begin{aligned}
\left(q_{2} x\right) \cup\left(q_{2} \omega_{0}\right) & =q_{2} x \cup q_{2} x=q_{2}(x \otimes x) H\left(f_{M}^{Q}\right) \\
& =(x \otimes \mathbb{Z} / 2)\left(A \otimes q_{2} \Sigma\right)\left(f_{M}^{Q}\right)=(x \otimes \mathbb{Z} / 2)(\sigma(\nu))
\end{aligned}
$$

where in the first equation we use $\omega_{0} \in{ }^{\#} A$ in (9.2) with $\omega=\omega_{0} \otimes 1$. This shows that $\sigma(\nu)$ is in fact the Poincaré dual of $\omega$.

We use diagram (3) also in the following computation of the group $\mathcal{J}$ in (8.28). The group $\operatorname{Hom}(A, A \otimes \mathbb{Z} / 2)$ in (8.28) is generated by the compositions

$$
\xi=y q_{2} x: A \rightarrow \mathbb{Z} \rightarrow \mathbb{Z} / 2 \rightarrow A \otimes \mathbb{Z} / 2
$$

with $x \in \# A$ and $y \in \operatorname{Hom}(\mathbb{Z} / 2, A \otimes \mathbb{Z} / 2)$. By Poincaré duality each element $z \in \mathbb{Z} \otimes A=A$ is of the form (see (5))

$$
z=(x \otimes A) H f_{M}^{Q}
$$

We now get the following equations where we compute the generators in $\mathcal{J}$, see (8.28).

$$
\begin{aligned}
P(A \otimes \xi) H\left(f_{M}\right) & =P\left(A \otimes y q_{2}\right)(A \otimes x) H f_{M} \\
& =P\left(A \otimes y q_{2}\right)(x \otimes A) H f_{M}^{Q} \\
& =P\left(A \otimes y q_{2}\right)(z) .
\end{aligned}
$$

On the other hand we get

$$
\left(A \otimes \eta_{n_{*}}\right)(\xi \otimes \Sigma)\left(f_{M}\right)=\left(A \otimes \eta_{n_{*}}\right)(y \otimes \mathbb{Z} / 2)\left(q_{2} x \otimes q_{2} \Sigma\right) f_{M}^{Q}
$$


where $\eta_{n_{*}}: \mathbb{Z} / 2=\mathbb{Z} / 2 \otimes \mathbb{Z} / 2^{r+1} \rightarrow \Pi_{2 n}\left\{S^{n}\right\}$ is given by (8.27). By (3) and (6) we see that

$$
\begin{aligned}
\left(q_{2} x \otimes q_{2} \Sigma\right) f_{M}^{Q} & =q_{2} x \cup q_{2} \omega_{0} \\
& =\left(q_{2} x \otimes q_{2} \omega_{0}\right) H\left(f_{M}^{Q}\right) \\
& =\left(q_{2} \otimes q_{2} \omega_{0}\right)(x \otimes A) H\left(f_{M}^{Q}\right) \\
& =\left(q_{2} \otimes q_{2} \omega_{0}\right)(z) .
\end{aligned}
$$

Here we also use (5) and (8). Thus (9), (10) and (11) show that $\mathcal{J}$ in (8.28) is generated by the elements

$$
\left\{\begin{array}{l}
\left(A \otimes \eta_{2 n-1}^{*}\right) f_{M} \\
P\left(y q_{2} \otimes A\right)(z)+\left(A \otimes \eta_{n_{*}}\right)\left(y q_{2} \otimes \omega\right)(z)=R
\end{array}\right.
$$

where $y \in \operatorname{Hom}(\mathbb{Z} / 2, A \otimes \mathbb{Z} / 2), z \in \mathbb{Z} \otimes A$. We clearly have $q_{2} \omega_{0}=\omega_{M}=\omega \in$ $\operatorname{Hom}(A, \mathbb{Z} / 2)$ for $\omega$ in (9.5). The operator $P$ in (12) is defined for $A \otimes \Pi_{2 n}\left\{S^{n}\right\}$ as in (8.25). Here we have

$$
\begin{aligned}
& \Pi_{2 n}\left\{S^{n}\right\}= \begin{cases}\mathbb{Z}^{P} \otimes \mathbb{Z} / 2 & \text { for } n=4, \\
\left(\mathbb{Z}^{P} \otimes \mathbb{Z} / 2\right) \oplus(\mathbb{Z} / 2 \oplus \mathbb{Z} / 2) & \text { for } n=8,\end{cases} \\
& \mathbb{Z}^{P} \otimes \mathbb{Z}_{2}=(\mathbb{Z} / 2 \oplus \mathbb{Z} / 2 \stackrel{(0,1)}{\rightarrow} \mathbb{Z} / 2 \stackrel{(1,0)}{\rightarrow} \mathbb{Z} / 2 \oplus \mathbb{Z} / 2) .
\end{aligned}
$$

The isomorphism (13) carries the bases elements $1 \oplus 0,0 \oplus 1 \in \mathbb{Z} / 2 \oplus \mathbb{Z} / 2=$ $\left(\mathbb{Z}^{P} \otimes \mathbb{Z} / 2\right)_{e}$ to $S \nu^{\prime} \eta_{7}$, resp. $\nu_{4} \eta_{7}$ for $n=4$ and to $S \sigma^{\prime} \eta_{15}$, resp. $\sigma_{8} \eta_{15}$ for $n=8$. Here we have $\left[\iota_{4}, \iota_{4}\right] \eta_{7}=S \nu^{\prime} \eta_{7}$ and $\left[\iota_{8}, \iota_{8}\right] \eta_{15}=S \sigma^{\prime} \eta_{15}$ and $H \nu_{4} \eta_{7}=\eta_{7}, H \sigma_{8} \eta_{15}=\eta_{15}$. (See Proposition 2.2, Lemma 5.4, p. 43, 5.11, Lemma 5.14, p. 63 in Toda's book [39].) For $n=8$ the generators of the summand $\mathbb{Z} / 2 \oplus \mathbb{Z} / 2$ in (13) are $\varepsilon_{8}$ and $\bar{\nu}_{8}$. This description of generators shows that we get $\eta_{2 n-1}^{*}$ by the composition in $\mathrm{QM}(Z)$

$$
\eta_{2 n-1}^{*}: \Pi_{2 n-1}\left\{S^{n}\right\} \stackrel{q_{2}}{\rightarrow} \Pi_{2 n-1}\left\{S^{n}\right\} \otimes \mathbb{Z} / 2=\mathbb{Z}^{P} \otimes Z / 2 \subset \Pi_{2 n}\left\{S^{n}\right\}
$$

where we use (1) and the inclusion given by (13). On the other hand we obtain

$$
\eta_{n_{*}}: \mathbb{Z} / 2=\mathbb{Z} / 2 \otimes \Sigma \Pi_{2 n-1}\left(S^{n}\right) \rightarrow \Pi_{2 n} S^{n}= \begin{cases}(\mathbb{Z} / 2)^{2}, & n=4, \\ (\mathbb{Z} / 2)^{4}, & n=8,\end{cases}
$$

as follows. We observe that $1 \otimes \nu_{5}$ generates $\mathbb{Z} / 2 \otimes \Sigma \Pi_{7} S^{4}$ and $\eta_{4_{*}}$ carries this element to

$$
\eta_{4_{*}}\left(1 \otimes \nu_{5}\right)=\eta_{4} \nu_{5}=S \nu^{\prime} \eta_{7}=1 \oplus 0 \in(\mathbb{Z} / 2)^{2}
$$

(by p. $44,5.9$ in Toda [39]). Morever $1 \otimes \sigma_{9}$ generates $\mathbb{Z} / 2 \otimes \Sigma \Pi_{15} S^{8}$ and $\eta_{8 *}$ carries this generator to (see 7.4 in Toda [39])

$$
\begin{aligned}
\eta_{8_{*}}\left(1 \otimes \sigma_{9}\right) & =\eta_{8} \sigma_{9}=S \sigma^{\prime} \eta_{15}+\overline{\nu_{8}}+\varepsilon_{8} \\
& =1 \oplus 0 \oplus 1 \oplus 1 \in(\mathbb{Z} / 2)^{4} .
\end{aligned}
$$

Hence we get for $\eta_{n_{*}}$ in (15) the formula

$$
\eta_{n_{*}}= \begin{cases}(1,0) & \text { for } n=4 \\ (1,0,1,1) & \text { for } n=8\end{cases}
$$


Using (13) we see that

$$
A \otimes \Pi_{2 n}\left\{S^{n}\right\}= \begin{cases}P(A) \otimes \mathbb{Z} / 2 & \text { for } n=4, \\ P(A) \otimes \mathbb{Z} / 2 \oplus(A \otimes \mathbb{Z} / 2)^{2} & \text { for } n=8 .\end{cases}
$$

Moreover the inclusion (14) shows that the first element in (12) corresponds to

$$
\left(A \otimes \eta_{2 n-1}^{*}\right) f_{M}=\nu \in P(A) \otimes \mathbb{Z} / 2 \subset A \otimes \Pi_{2 n}\left\{S^{n}\right\} .
$$

On the other hand the second element in (12) can be described as follows. We first deal with the case $n=4$. Then we have the commutative diagram

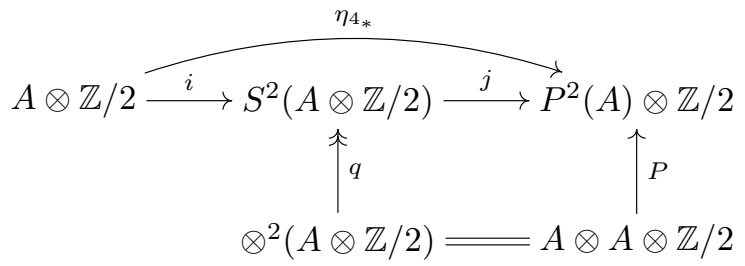

where $j i=\eta_{4_{*}}$ since $a \otimes P_{n}=[a, a] \otimes n$ by $(8.2)(4)$ so that $\eta_{4_{*}}(a \otimes 1)=a \otimes \eta_{4_{*}} 1=$ $a \otimes P 1=[a, a] \otimes 1=j(a \cdot a)=j i(a)$. The quotient map $q$ carries $x \otimes y$ to the equivalence class $x \cdot y=\{x \otimes y\}$ with $j(x \cdot y)=[x, y] \otimes 1=P(x \otimes y \otimes 1)$. Now the second element in (12) coincides with the following element $R$ where $y, z \in A \otimes \mathbb{Z} / 2$ correspond to the elements $y, z$ in (12).

$$
R=j\left(R^{\prime}\right) \quad \text { with } R^{\prime}=y \cdot z+i(y \cdot \omega(z)) .
$$

The elements $R^{\prime}$ generate the kernel of $\omega_{*}$ in (9.4)(3). Hence we obtain by (20) the formula

$$
\mathcal{E}(M \mid \dot{M})=E_{4}(\omega) / \bar{\omega}_{*}(\nu) \text { for } n=4 .
$$

A similar argument yields the result for $n=8$. In this case we get by (18), see $(9.4)(4)$,

$$
R=j(y \cdot z+i(y \cdot \omega(z))) \oplus y \cdot \omega(z) \oplus y \cdot \omega(z) .
$$

These elements $R$ generate the kernel of $\bar{\omega}_{*}$ in $(9.4)(4),(5)$ so that by (20) we get

$$
\mathcal{E}(M \mid \dot{M})=E_{8}(\omega) / \bar{\omega}_{*}(\nu) \text { for } n=8 .
$$

This completes the proof of Theorem (9.5).

\section{REMARK ON DIFFEOMORPHISMS}

Let $M$ be a differential manifold and let $\Pi_{0} \operatorname{Diff}_{+}(M)$ be the group of isotopy classes of orientation preserving diffeomorphisms of $M$. Then we have the homomorphism

$$
\Psi: \Pi_{0} \operatorname{Diff}_{+}(M) \rightarrow \mathcal{E}_{+}(M)
$$

which carries an isotopy class of a diffeomorphism $h$ to the homotopy class of $h$. Here $\mathcal{E}_{+}(M)$ is the subgroup of orientation preserving homotopy equivalences in $\mathcal{E}(M)$. We use the invariants $\alpha_{M}$ and $\delta_{M}$ in (8.10) and for $\alpha=\alpha_{M}$ or $\alpha=\delta_{M}$ let Aut $_{+}(\alpha)$ be the subgroup of $\operatorname{Aut}(\alpha)$ consisting of elements $x$ with $\operatorname{deg}(x)=+1$. 
By our result (8.14) we get the following theorem on the homomorphism $\Psi$ above. In the theorem we use the diagram

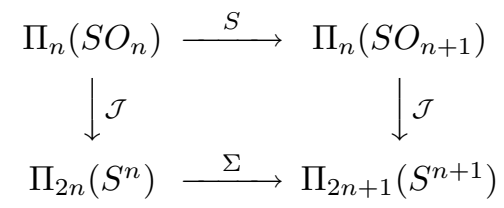

where $\mathcal{J}$ is the $\mathcal{J}$-homomorphism and where $S$ is induced by the inclusion $S O_{n} \subset$ $S O_{n+1}$. Since the diagram commutes up to sign we get the well defined homomorphism

$$
\mathcal{J}: S \Pi_{n}\left(S O_{n}\right) \rightarrow \Sigma \Pi_{2 n}\left(S^{n}\right)=\text { Tor } \Pi_{2 n+1}\left(S^{n+1}\right)
$$

needed in the following result:

(10.3) Theorem. Let $M$ be a differential $(n-1)$-connected $2 n$-manifold, $n \geq 3$, which is almost parallelizable. Then there is a commutative diagram in which the columns are short exact, $A=H_{n} M$.

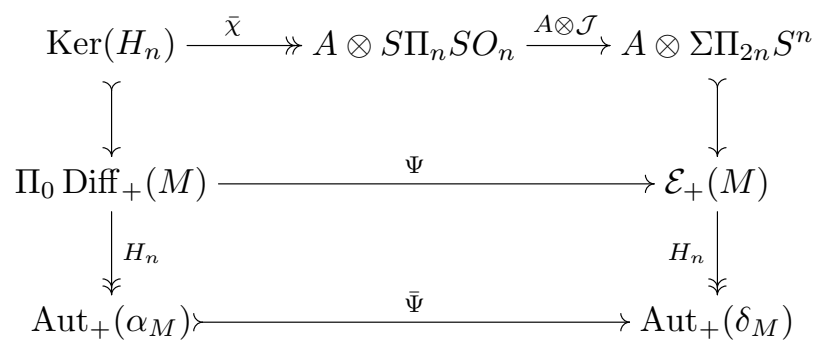

Here $H_{n}$ is given by the homology functor and the inclusion $\bar{\Psi}$ is surjective if $\mathcal{J}_{n}$ in (8.7) is injective, i.e. for $n$ odd or $n \equiv 6$ (8). Moreover $\bar{\chi}$ is surjective with $\operatorname{kernel}(\bar{\chi}) \cong \Theta_{2 n+1} /\left\{\Sigma_{M}\right\}$ where $\Sigma_{M}$ is an element in the group of $(2 n+1)$ dimensional homotopy spheres $\Theta_{2 n+1}$ of order 2 and depending only on $M$.

The homomorphism $\bar{\chi}$ is obtained by the homomorphism $\chi$ in [30] via Poincaré duality; commutativity of the diagram follows from the definition of $\chi$ and the definition of $\mathcal{J}$; see the proof of Wall of Lemma 8 [40].

(10.4) Corollary. If $\mathcal{J}$ in (10.2) is injective then we have $\operatorname{Ker} \Psi=\operatorname{Ker} \bar{\chi}=$ $\Theta_{2 n+1} /\left\{\Sigma_{M}\right\}$. Moreover if $\mathcal{J}$ in (10.2) is surjective and $n$ odd or $n \equiv 6$ (8) then $\Psi$ is surjective. On the other hand if $\mathcal{J}=0$ in (10.2) and if $n$ is odd or $n \equiv 6$ (8) then the fundamental extension for $\mathcal{E}(M)$ has a splitting induced by $\Psi$.

(10.5) Remark. We would like to warn the reader that the paper of Kolosov concerning $\Psi$ in (10.3) (see Math. USSR-Sb. 41 (1982)) contains various errors, in particular his main result Theorem 1 contradicts results of Kreck [30] and Theorem (10.3) above.

\section{REFERENCES}

1. J. F. Adams, On the groups $J(X)$. IV, Topology 5 (1966), 21-27. MR 33:6628

2. M. Arkowitz, The group of self-homotopy equivalences - a survey, Lecture Notes in Math., vol. 1425, Springer, 1990, pp. 170-203. MR 91i:55001

3. M. F. Atiyah, Thom complexes, Proc. London Math. Soc. (3) 11 (1961), 291-310. MR 24:A1727 
4. M. Aubry and J.-M. Lemaire, Sur certaines équivalences d'homotopies, Ann. Inst. Fourier (Grenoble) 41 (1991), 173-187. MR 92d:55010

5. H. J. Baues, Obstruction theory, Lecture Notes in Math., vol. 628, Springer-Verlag, 1977, 387 pages. MR 57:7600

6. (1980), 171 pages. MR 81m:55010

7. Commutator calculus and groups of homotopy classes, London Math. Society, Lecture Notes Series, 50, 1981, 160 pages. MR 83b:55012

8. _ Algebraic homotopy, Cambridge Studies in Advanced Math., 15, Cambridge Univ. Press, 1989, 450 pages. MR 90i:55016

9. Combinatorial homotopy and 4-dimensional complexes, De Gruyter Expositions in Math., De Gruyter, 1991, 380 pages. MR 92h:55008

10. _ Whitehead Produkte und Hindernisse in dem Produkt von Abbildungskegeln, Arch. Math. 25 (1974), 184-197. MR 49:11509

11. - On homotopy classification problems of J. H. C. Whitehead, Algebraic Topology, Lecture Notes in Math., vol. 1172, Springer, 1985, 17-55. MR 87f:55006

12. L Metastable homotopy and homology, Quaestiones Math. 14 (1991), 161-178. MR 92j:55012

13. _ Quadratic functors and metastable homotopy, J. Pure Appl. Algebra 91 (1994), 49107. MR 94j:55022

14. - On the homotopy groups of mapping cones, Appendix of "Homotopy Type and Homology", Oxford Math. Monographs, Oxford Univ. Press, 1996, 489 pages.

15. H. J. Baues and J. Buth, On the group of homotopy equivalences of simply connected 5dimensional manifolds, Math. Z. (to appear).

16. R. Bott, The stable homotopy of the classical groups, Ann. of Math. (2) 70 (1959), 313-337. MR 22:987

17. G. Cooke, Replacing homotopy actions by topological actions, Trans. Amer. Math. Soc. 237 (1978), 391-406. MR 57:1529

18. T. D. Cochran and N. Habegger, On the homotopy theory of simply connected four manifolds, Topology 29 (1990), 419-440. MR 91h:57006

19. W. Dreckmann, Die Relationen der Homotopieoperationen, Diplomarbeit Bonn, 1987.

20. S. Eilenberg and S. Mac Lane, On the groups H(П,n). II, Ann. of Math. (2) 60 (1954), 49-139. MR 16:391a

21. M. H. Freedman, The topology of four-dimensional manifolds, J. Differential Geometry $\mathbf{1 7}$ (1982), 357-453. MR 84b:57006

22. I. M. James, The suspension triad of a sphere, Ann. of Math. (2) 63 (1956), 407-429. MR 18:58f

23. _ Spaces associated with Steifel manifolds, Proc. London Math. Soc. (3) 9 (1959), 115-140. MR 21:1596

24. P. J. Hilton, Homotopy theory and duality, Gordon-Breach, 1965. MR 33:6624

25. F. Hirzebruch, W. Neumann and S. S. Koh, Differentiable manifolds and quadratic forms, Lecture Notes in Pure and Appl. Math., 4, Marcel Dekker, 1971. MR 49:6250

26. P. J. Kahn, Self-equivalences of $(n-1)$-connected $2 n$-manifolds, Bull. Amer. Math. Soc. 72 (1966), 562-566. MR 32:8346

27. _ Self-equivalences of $(n-1)$-connected 2n-manifolds, Math. Ann. 180 (1969), 26-47. MR 39:6344

28. A. M. Kervaire and J. W. Milnor, Group of homotopy spheres. I, Ann. of Math. (2) 77 (1963), 504-537. MR 26:5584

29. A. M. Kervaire, Some non-stable homotopy groups of Lie groups, Illinois J. Math. 4 (1960), 161-169. MR 22:4075

30. M. Kreck, Isotopy classes of diffeomorphisms of $(k-1)$-connected almost-parallelizable $2 k$ manifolds, (Algebraic Topology Aarhus 1978), Lecture Notes in Math., vol. 763, Springer, 1979, pp. 643-650. MR 81i:57029

31. M. E. Mahowald, Some remarks on the Kervaire invariant problem from the homotopy point of view, Algebraic Topology, Proc. Sympos. Pure Math., vol. 22, Amer. Math. Soc., Providence, R.I., 1970. MR 48:1245.

32. J. W. Milnor and I. D. Stasheff, Characteristic classes, Ann. of Math. Stud., no. 76, Princeton Univ. Press, 1974. MR 55:13428 
33. W. Metzler and A. Zimmermann, Selbstäquivalenzen von $S^{3} \times S^{3}$ in quaterionischer Behandlung, Arch. Math. 22 (1971), 209-213. MR 45:8786

34. F. Quinn, Isotopy of 4-manifolds, J. Differential Geometry 24 (1986), 343-372. MR 88f:57020

35. N. Sawashita, On the group of self-equivalences of the product of spheres, Hiroshima Math. J. 5 (1975), 69-86. MR 51:14035

36. E. H. Spanier, Algebraic topology, McGraw-Hill, 1966. MR 35:1007

37. M. Spivak, Spaces satisfying Poincaré duality, Topology 6 (1967), 77-101. MR 42:9099

38. R. Stöcker, Zur Topologie der Poincaré-Räume, Habilitationsschrift, Universität Bochum, 1974.

39. H. Toda, Composition methods in homotopy groups of spheres, Ann. of Math. Stud., no. 49, Princeton Univ. Press, 1962. MR 26:777

40. C. T. C. Wall, Classification of $(n-1)$-connected 2n-manifolds, Ann. of Math. (2) 75 (1962), 163-189. MR 26:5570

41. J. H. C. Whitehead, A certain exact sequence, Ann. of Math. (2) 52 (1950), 51-110. MR 12: $43 \mathrm{C}$

42. W. D. Barcus and M. G. Barratt, On the homotopy classification of a fixed map, Trans. Amer. Math. Soc. 88 (1958), 57-74. MR 20:3540

43. S. Oka, N. Sawashita and M. Sugawara, On the group of self-equivalences of a mapping cone, Hiroshima Math. J. 4 (1974), 9-28. MR 49:11503

44. J. W. Rutter, A homotopy classification of maps into an induced fibre space, Topology 6 (1967), 379-403. MR 35:4922

Max Planck Institute for Mathematics, Gottfried-Claren-Strasse 26, 53225 Bonn, GERMANY

E-mail address: baues@mpim-bonn.mpg.de 